\title{
Three-dimensional imaging of the nucleon and semi-inclusive high-energy reactions
}

\author{
Kai-Bao Chen, Shu-Yi Wei, Zuo-Tang Liang ${ }^{\dagger}$ \\ School of Physics 83 Key Laboratory of Particle Physics and Particle Irradiation (Ministry of Education), \\ Shandong University, Jinan 250100, China \\ Corresponding author. E-mail: †liang@sdu.edu.cn \\ Received May 12, 2015; accepted June 24, 2015
}

\begin{abstract}
We present a short overview of studies of the transverse-momentum-dependent parton distribution functions of the nucleon. The aim of such studies is to provide three-dimensional imaging of the nucleon and a comprehensive description of semi-inclusive high-energy reactions. By summarizing what we have done in constructing the theoretical framework for inclusive deep inelastic lepton-nucleon scattering and one-dimensional imaging of the nucleon, we try to sketch out an outline of what we need to do to construct such a comprehensive theoretical framework for semi-inclusive processes in terms of three-dimensional gauge-invariant parton distributions. Next, we present an overview of what we have already achieved, with an emphasis on the theoretical framework for semi-inclusive reactions in leading-order perturbative quantum chromodynamics but with leading and higher twist contributions. We summarize in particular the results for the differential cross section and azimuthal spin asymmetries in terms of the gauge-invariant transverse-momentum-dependent parton distribution functions. We also briefly summarize the available experimental results on semi-inclusive reactions and the parameterizations of transverse-momentum-dependent parton distributions extracted from them and present an outlook for future studies.
\end{abstract}

Keywords transverse momentum dependence, parton distribution function, fragmentation function, collinear expansion, higher twists

PACS numbers 12.38.-t, 12.38.Bx, 12.39.St, 13.60.-r, 13.66.Bc, 13.87.Fh, 13.88.+e, 13.40.-f, 13.85. Ni

\section{Contents}

1 Introduction

2 Inclusive DIS \& one-dimensional imaging of the nucleon

3 TMDs defined via quark-quark correlator

4 Accessing the TMDs in high-energy reactions

5 Available data and parameterizations

6 Summary and outlook

Acknowledgements

References and notes

\section{Introduction}

In the ongoing intensive study of the nucleon structure, three-dimensional imaging has become the frontier and has been a hot topic in recent years. It is commonly

*Special Topic: Spin Physics (Eds. Haiyan Gao \& Bo-Qiang Ma). recognized that three-dimensional imaging reveals much more abundant physics of the nucleon structure and the properties of quantum chromodynamics (QCD). The study of three-dimensional imaging was initially triggered by the experimental finding of striking singlespin asymmetries (SSAs) in inclusive hadron production in hadron-hadron collisions with transversely polarized hadrons [1]. Gradually it grew into a field aiming at a comprehensive three-dimensional description of the nucleon structure, including the spin and transverse momentum dependence.

One-dimensional imaging of the nucleon is provided by the parton distribution functions (PDFs) such as the number density $q(x)$, helicity distribution $\Delta q(x)$, and transversity $\delta q(x)$ for quarks of different flavors in the nucleon. These one-dimensional PDFs can be studied in inclusive high-energy reactions and are necessary for the description of such inclusive processes. The threedimensional case, in which the parton transverse momen- 
tum is also considered, involves not only direct extensions of these distribution functions to include the transverse momentum dependence, but also many other correlation functions that describe in particular the correlations between the transverse momenta and spins, such as the Sivers function, Boer-Mulders function, and pretzelocity. They are generally called transverse-momentumdependent (TMD) PDFs. Moreover, higher twist effects also become important and need to be considered consistently. The content of the studies is therefore much more abundant and more interesting. These TMD PDFs can be studied in semi-inclusive reactions and are necessary for the description of such processes.

The study of three-dimensional imaging of the nucleon is in the rapidly developing stage, and it is not easy to provide a comprehensive overview of all the different aspects of the studies. Here, we choose to arrange the review as follows: First, we will briefly review what we have done in constructing the theoretical framework in onedimensional case with inclusive deep inelastic leptonnucleon scattering (DIS). In this way, we hope that we can sketch out the main line of what we need to do in the three-dimensional case. Next, we will try to follow this line and summarize the progress already achieved along this direction and what we need to do next. This brief review of the one-dimensional case will be presented in Section 2. In Section 3, we will summarize the TMDs defined via the quark-quark correlator. In Section 4, we will present a brief overview of the available information for constructing the theoretical framework of semiinclusive processes. In Section 5, we will summarize the available experimental results and TMD parameterizations extracted from them. Finally, we will summarize this review in Section 6.

This overview article is an extended version of a plenary talk at the 21st International Symposium on Spin Physics (Spin2014) [2]. It is clear that the simplest and most basic picture is at the leading order (LO) in perturbative QCD (pQCD) and at the leading twist. Hence, there are also two major directions in theoretical developments toward a comprehensive description of semiinclusive processes: taking higher-order pQCD into account and considering higher twist contributions. These contributions are important not only for higher accuracy but also for consistency. The major advances that have been made in recent years have also followed one of these two directions, i.e., either at the leading twist but leading and higher order in PQCD or in the LO in pQCD but leading and higher twists. The talk [2] concentrated mainly on the second direction. For higher-order pQCD contributions involving the evolution of PDFs, an overview talk was presented by Daniel Boer at the same conference [3]. There are also many other reviews and monographs (e.g., $[4,6,7])$. The study of higher orders in $\mathrm{pQCD}$ and higher twists seems to be rather difficult, and even the factorization properties are unclear [5]. In this article, we follow the same line as in the talk [2] but briefly summarize progress in the studies on QCD evolution and refer interested readers to those reviews.

\section{Inclusive DIS \& one-dimensional imaging of the nucleon}

Our studies on the structure of a fast-moving nucleon started with inclusive DIS such as $e^{-}+N \rightarrow e^{-}+X$. We recall that, under the one-photon exchange approximation, the differential cross section is given by the Lorentz contraction of the well-known leptonic tensor $L^{\mu \nu}\left(l, l^{\prime}, \lambda_{l}\right)$ and the hadronic tensor $W_{\mu \nu}(q, p, S)$, i.e.,

$$
\mathrm{d} \sigma=\frac{2 \alpha_{\mathrm{em}}^{2}}{s Q^{4}} L^{\mu \nu}\left(l, l^{\prime}, \lambda_{l}\right) W_{\mu \nu}(q, p, S) \frac{\mathrm{d}^{3} l^{\prime}}{2 E_{l^{\prime}}} .
$$

The leptonic tensor is calculable and is given by

$$
L_{\mu \nu}\left(l, l^{\prime}, \lambda_{l}\right)=2\left(l_{\mu} l_{\nu}^{\prime}+l_{\nu} l_{\mu}^{\prime}-g_{\mu \nu} l \cdot l^{\prime}\right)+\mathrm{i} 2 \lambda_{l} \epsilon_{\mu \nu \rho \sigma} l^{\rho} q^{\sigma} .
$$

Information on the structure of the nucleon is contained in the hadronic tensor, which is defined as

$$
\begin{aligned}
W_{\mu \nu}(q, p, S)= & \frac{1}{2 \pi} \sum_{X}\left\langle p, S\left|j_{\mu}(0)\right| X\right\rangle\left\langle X\left|j_{\nu}(0)\right| p, S\right\rangle \\
& \times(2 \pi)^{4} \delta^{4}\left(p+q-p_{X}\right) .
\end{aligned}
$$

Here, $l$ and $p$ denote the four-momenta of the lepton and nucleon, respectively, and primes indicate the final states; $\lambda$ stands for the helicity, and $S$ is the polarization vector of the nucleon. We use light-cone coordinates and define the light-cone unit vectors as $\bar{n}=\left(1,0, \overrightarrow{0}_{\perp}\right)$, $n=\left(0,1, \overrightarrow{0}_{\perp}\right)$, and $n_{\perp}=\left(0,0, \vec{n}_{\perp}\right)$, so a general fourvector can be decomposed as $A^{\mu}=A^{+} \bar{n}^{\mu}+A^{-} n^{\mu}+A_{\perp}^{\mu}$, where $A^{ \pm}=\left(A^{0} \pm A^{3}\right) / \sqrt{2}$, and $A_{\perp}=\left(0,0, \vec{A}_{\perp}\right)$. We work in the center-of-mass frame of $\gamma^{*} N$ and choose the nucleon's momentum as being in the $z$ direction, so $p$ and $S$ are decomposed as

$$
\begin{aligned}
& p^{\mu}=p^{+} \bar{n}^{\mu}+\frac{M^{2}}{2 p^{+}} n^{\mu}, \\
& S^{\mu}=\lambda \frac{p^{+}}{M} \bar{n}^{\mu}+S_{T}^{\mu}-\lambda \frac{M}{2 p^{+}} n^{\mu} .
\end{aligned}
$$

The Bjorken variable is defined as $x_{B}=Q^{2} /(2 p \cdot q), q=$ $-x_{B} p^{+} \bar{n}+n Q^{2} /\left(2 x_{B} p^{+}\right)$; we also define $y=(p \cdot q) /(p \cdot l)$.

The theoretical framework for inclusive DIS has been constructed in the following steps. First, we studied the 
kinematics and obtained the general form of the hadronic tensor by applying the basic constraints from the general symmetry requirements such as Lorentz covariance, gauge invariance, parity conservation, and Hermiticity, e.g.,

$$
\begin{aligned}
& q^{\mu} W_{\mu \nu}(q, p, S)=0, \\
& W_{\mu \nu}(\tilde{q}, \tilde{p},-\tilde{S})=W^{\mu \nu}(q, p, S), \\
& W_{\mu \nu}^{*}(q, p, S)=W_{\nu \mu}(q, p, S),
\end{aligned}
$$

where $\tilde{A}$ denotes the results of $A$ after space reflection, i.e., $\tilde{A}^{\mu}=A_{\mu}$. The general form of the hadronic tensor is given by the sum of a symmetric part and an antisymmetric part,

$$
W_{\mu \nu}(q, p, S)=W_{\mu \nu}^{(S)}(q, p)+\mathrm{i} W_{\mu \nu}^{(A)}(q, p, S)
$$

where $W_{\mu \nu}^{(S)}(q, p)$ and $W_{\mu \nu}^{(A)}(q, p, S)$ are given by

$$
\begin{aligned}
& W_{\mu \nu}^{(S)}(q, p)=2\left(-g_{\mu \nu}+\frac{q_{\mu} q_{\nu}}{q^{2}}\right) F_{1}\left(x, Q^{2}\right) \\
& +\frac{1}{x Q^{2}}\left(q_{\mu}+2 x p_{\mu}\right)\left(q_{\nu}+2 x p_{\nu}\right) F_{2}\left(x, Q^{2}\right), \\
& W_{\mu \nu}^{(A)}(q, p, S)=\frac{2 M \varepsilon_{\mu \nu \rho \sigma} q^{\rho}}{p \cdot q} \\
& \quad \times\left[S^{\sigma} g_{1}\left(x, Q^{2}\right)+\left(S^{\sigma}-\frac{S \cdot q}{p \cdot q} p^{\sigma}\right) g_{2}\left(x, Q^{2}\right)\right],
\end{aligned}
$$

respectively. We found that the hadronic tensor is determined by four independent structure functions, $F_{1}, F_{2}$, $g_{1}$, and $g_{2}$, where the first two describe the unpolarized case, and the latter two are needed for polarized cases.

Our knowledge of one-dimensional imaging of the nucleon starts with the "intuitive parton model", which is very nicely formulated, e.g., in Ref. [8]. Here, it was argued that, in a fast-moving frame, because of time dilation, quantum fluctuations such as vacuum polarizations can exist for quite a long time. In the infinite momentum frame, such fluctuations exist forever. In this case, a fast-moving nucleon can be viewed as a beam of free "partons". The probability of the scattering of an electron with a nucleon is taken as the incoherent sum of that of the scattering with each individual parton, more precisely, as a convolution of the number density of partons in the nucleon with the probability of scattering with the parton, i.e.,

$$
|\mathcal{M}(e N \rightarrow e X)|^{2}=\sum_{q} \int \mathrm{d} x f_{q}(x)|\hat{\mathcal{M}}(e q \rightarrow e q)|^{2},
$$

where $f_{q}(x)$ is the number density of partons of flavor $q$ in the nucleon. In this way, we obtained the famous results [8]

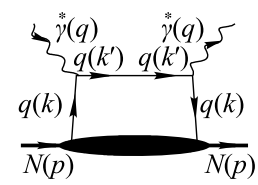

(a)

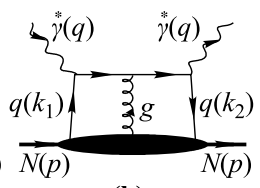

(b)

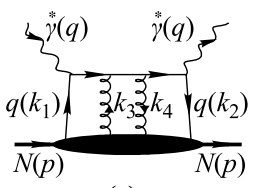

(c)
Fig. 1 Examples of the Feynman diagram series with multiple gluon scattering considered for $\gamma^{*}+N \rightarrow q+X$ with (a) $j=0$, (b) $j=1$ and (c) $j=2$ gluons exchanged.

$$
\begin{aligned}
& F_{2}\left(x, Q^{2}\right)=2 x F_{1}\left(x, Q^{2}\right)=\sum_{q} e_{q}^{2} x f_{q}(x), \\
& g_{1}\left(x, Q^{2}\right)=\sum_{q} e_{q}^{2} \Delta f_{q}(x), \\
& g_{1}\left(x, Q^{2}\right)+g_{2}\left(x, Q^{2}\right)=\sum_{q} e_{q}^{2} x \delta f_{q}(x) .
\end{aligned}
$$

Here, we would like to point out that, with this intuitive parton model, we are doing nothing else but the impulse approximation that we often use in describing a collision process, where we make the following approximations:

- during the interaction of the electron with the parton, interactions between the partons are neglected;

- the electron interacts with only one single parton each time;

- the scatterings of the electron with different partons are added incoherently.

Although the physical picture of the intuitive model is very clear, and the model is elegant and practical, we are not satisfied with the formulation because it is partly qualitative or semiclassical; hence, it is not easy to control the accuracy. A proper formulation should be based on quantum field theory (QFT) and is obtained by starting with the Feynman diagram, as shown in Fig. 1(a). From this diagram, we obtain immediately that

$$
W_{\mu \nu}^{(0)}(q, p, S)=\frac{1}{2 \pi} \int \frac{\mathrm{d}^{4} k}{(2 \pi)^{4}} \operatorname{Tr}\left[\hat{H}_{\mu \nu}^{(0)}(k, q) \hat{\phi}^{(0)}(k, p, S)\right],
$$

where $k$ is the four-momentum of the parton.

$$
\hat{H}_{\mu \nu}^{(0)}(q, k)=\gamma_{\mu}(\not k+\not q) \gamma_{\nu}(2 \pi) \delta_{+}\left((k+q)^{2}\right)
$$

is a calculable hard part. The matrix element

$$
\hat{\phi}^{(0)}(k, p, S)=\int \mathrm{d}^{4} z \mathrm{e}^{\mathrm{i} k \cdot z}\langle p, S|\bar{\psi}(0) \psi(z)| p, S\rangle
$$

is known as the quark-quark correlator and describes the structure of the nucleon. By taking the collinear approximation, i.e., taking $k \approx x p$, and neglecting the powersuppressed contributions, i.e., the $o(M / Q)$ terms, we obtain 


$$
\begin{aligned}
W_{\mu \nu}^{(0)}(q, p) & \approx\left[\left(-g_{\mu \nu}+\frac{q_{\mu} q_{\nu}}{q^{2}}\right)+\frac{(q+2 x p)_{\mu}(q+2 x p)_{\nu}}{2 x p \cdot q}\right] \\
& \times f_{q}(x) .
\end{aligned}
$$

This is exactly the same result as that obtained from Eq. (2.12) on the basis of the intuitive parton model. At the same time, we obtain the QFT operator expression of $f_{q}(x)$, defined via the quark-quark correlator given by Eq. (2.18), as

$$
f_{q}(x)=\int \frac{\mathrm{d} z^{-}}{2 \pi} \mathrm{e}^{\mathrm{i} x p^{+} z^{-}}\left\langle p\left|\bar{\psi}(0) \frac{\gamma^{+}}{2} \psi(z)\right| p\right\rangle .
$$

By inserting the expanded expression for the field operator $\psi(z)$ in terms of the plane wave and the creation and/or annihilation operators, we see clearly that $f_{q}(x)$ is indeed the number density of partons in the nucleon. However, from this expression, we also immediately see a severe problem; i.e., this expression is not (local) gauge invariant! We understand that the physical quantity has to be gauge invariant and therefore have to find a solu-

tion for this.

The gauge-invariant formulation is obtained by taking into account the multiple gluon scattering shown by the diagram series in Figs. 1(a)-(c). This is clear because (local) gauge invariance implies the existence of a gauge interaction that needs to be taken into account. In this way, we obtain

$$
W_{\mu \nu}(q, p, S)=\sum_{j=0}^{\infty} W_{\mu \nu}^{(j)}(q, p, S),
$$

where $W_{\mu \nu}^{(j)}(q, p, S)$ represents the contribution from the diagram with exchange of $j$ gluon(s). They are all expressed as a trace of a calculable hard part and a matrix element depending on the structure of the nucleon. E.g., corresponding to Fig. 1(b), we have $j=1$, and $W_{\mu \nu}^{(1)}(q, p, S)$ is given by

$$
W_{\mu \nu}^{(1)}(q, p, S)=\sum_{c=L, R} W_{\mu \nu}^{(1, c)}(q, p, S)
$$

$$
\hat{\phi}_{\rho}^{(1)}\left(k_{1}, k_{2}, p, S\right)=\int \mathrm{d}^{4} z \mathrm{~d}^{4} y \mathrm{e}^{\mathrm{i} k_{1} z+\left(k_{2}-k_{1}\right) y}\left\langle p, S\left|\bar{\psi}(0) A_{\rho}(y) \psi(z)\right| p, S\right\rangle,
$$

where $c$ in the superscript represents different cuts (left or right) in the diagram. Similarly, corresponding to Fig. $1(\mathrm{c})$, we have

$$
\begin{aligned}
& W_{\mu \nu}^{(2)}(q, p, S)=\sum_{c=L, M, R} W_{\mu \nu}^{(2, c)}(q, p, S), \\
& W_{\mu \nu}^{(2, c)}(q, p, S)=\frac{1}{2 \pi} \int \frac{\mathrm{d}^{4} k_{1}}{(2 \pi)^{4}} \frac{\mathrm{d}^{4} k_{2}}{(2 \pi)^{4}} \frac{\mathrm{d}^{4} k}{(2 \pi)^{4}} \operatorname{Tr}\left[\hat{H}_{\mu \nu}^{(2, c) \rho \sigma}\left(k_{1}, k_{2}, k, q\right) \hat{\phi}_{\rho \sigma}^{(2)}\left(k_{1}, k_{2}, k, p, S\right)\right], \\
& \hat{\phi}_{\rho \sigma}^{(2)}\left(k_{1}, k_{2}, k, p, S\right)=\int \mathrm{d}^{4} y \mathrm{~d}^{4} y^{\prime} \mathrm{d}^{4} z \mathrm{e}^{\mathrm{i} k_{1} y+\mathrm{i} k\left(y^{\prime}-y\right)+\mathrm{i} k_{2}\left(z-y^{\prime}\right)}\left\langle p, S\left|\bar{\psi}(0) g A_{\rho}(y) g A_{\sigma}\left(y^{\prime}\right) \psi(z)\right| p, S\right\rangle .
\end{aligned}
$$

The matrix element is now a quark- $j$-gluon(s)-quark correlator. We also immediately see that none of these quark- $j$-gluon(s)-quark correlators is gauge invariant.

To obtain the gauge-invariant form, we need to ap- ply the collinear expansion proposed in [9-11], which is carried out in the following four steps.

(1) Make Taylor expansions of all the hard parts at $k_{i}=x_{i} p$, e.g.,

$$
\begin{aligned}
& \hat{H}_{\mu \nu}^{(0)}(k, q)=\hat{H}_{\mu \nu}^{(0)}(x)+\frac{\partial \hat{H}_{\mu \nu}^{(0)}(x)}{\partial k_{\rho}} \omega_{\rho}^{\rho^{\prime}} k_{\rho^{\prime}}+\frac{1}{2} \frac{\partial^{2} \hat{H}_{\mu \nu}^{(0)}(x)}{\partial k_{\rho} \partial k_{\sigma}} \omega_{\rho}^{\rho^{\prime}} k_{\rho^{\prime}} \omega_{\sigma}^{\sigma^{\prime}} k_{\sigma^{\prime}}+\cdots, \\
& \hat{H}_{\mu \nu}^{(1, L) \rho}\left(k_{1}, k_{2}, q\right)=\hat{H}_{\mu \nu}^{(1, L) \rho}\left(x_{1}, x_{2}\right)+\frac{\partial \hat{H}_{\mu \nu}^{(1, L) \rho}\left(x_{1}, x_{2}\right)}{\partial k_{1 \sigma}} \omega_{\sigma}^{\sigma^{\prime}} k_{1 \sigma^{\prime}}+\frac{\partial \hat{H}_{\mu \nu}^{(1, L) \rho}\left(x_{1}, x_{2}\right)}{\partial k_{2 \sigma}} \omega_{\sigma}^{\sigma^{\prime}} k_{2 \sigma^{\prime}}+\cdots,
\end{aligned}
$$

and so on, where $\omega_{\rho}^{\rho^{\prime}}$ is a projection operator defined by $\omega_{\rho}^{\rho^{\prime}} \equiv g_{\rho}^{\rho^{\prime}}-\bar{n}_{\rho} n^{\rho^{\prime}}$.

(2) Decompose the gluon field into longitudinal and transverse components, i.e.,

$$
A_{\rho}(y)=A^{+}(y) \bar{n}_{\rho}+\omega_{\rho}^{\rho^{\prime}} A_{\rho^{\prime}}(y) .
$$

(3) Apply the Ward identities, such as

$$
\begin{aligned}
& \frac{\partial \hat{H}_{\mu \nu}^{(0)}(x)}{\partial k_{\rho}}=-\hat{H}_{\mu \nu}^{(1) \rho}(x, x), \\
& \frac{\partial \hat{H}_{\mu \nu}^{(1, L) \rho}\left(x_{1}, x_{2}\right)}{\partial k_{1, \sigma}}=-\hat{H}_{\mu \nu}^{(2, L) \rho \sigma}\left(x_{1}, x_{1}, x_{2}\right)
\end{aligned}
$$




$$
\begin{aligned}
& -\hat{H}_{\mu \nu}^{(2, M) \rho \sigma}\left(x_{1}, x_{1}, x_{2}\right), \\
& p_{\rho} \hat{H}_{\mu \nu}^{(1, L) \rho}\left(x_{1}, x_{2}\right)=\frac{H_{\mu \nu}^{(0)}\left(x_{1}\right)}{x_{2}-x_{1}-\mathrm{i} \epsilon}, \\
& p_{\rho} \hat{H}_{\mu \nu}^{(2, L) \rho \sigma}\left(x_{1}, x, x_{2}\right)=\frac{1}{x-x_{1}-\mathrm{i} \epsilon} H_{\mu \nu}^{(1, L) \sigma}\left(x_{1}, x_{2}\right), \\
& p_{\rho} \hat{H}_{\mu \nu}^{(2, M) \rho \sigma}\left(x_{1}, x, x_{2}\right)=-\frac{1}{x_{2}-x_{1}-\mathrm{i} \epsilon} H_{\mu \nu}^{(1, L) \sigma}\left(x_{1}, x_{2}\right) \\
& -\frac{1}{x_{1}-x+\mathrm{i} \epsilon} H_{\mu \nu}^{(1, R) \sigma}\left(x, x_{2}\right) . \\
& W_{\mu \nu}(q, p, S)=\sum_{j} \tilde{W}_{\mu \nu}^{(j)}(q, p, S), \\
& \tilde{W}_{\mu \nu}^{(0)}(q, p, S)=\frac{1}{2 \pi} \int \frac{\mathrm{d}^{4} k}{(2 \pi)^{4}} \operatorname{Tr}\left[\hat{H}_{\mu \nu}^{(0)}(x) \hat{\Phi}^{(0)}(k, p, S)\right], \\
& \tilde{W}_{\mu \nu}^{(1)}(q, p, S)=\frac{1}{2 \pi} \int \frac{\mathrm{d}^{4} k_{1}}{(2 \pi)^{4}} \frac{\mathrm{d}^{4} k_{2}}{(2 \pi)^{4}} \sum_{c=L, R} \operatorname{Tr}\left[\hat{H}_{\mu \nu}^{(1, c) \rho}\left(x_{1}, x_{2}\right) \omega_{\rho}^{\rho^{\prime}} \hat{\Phi}_{\rho^{\prime}}^{(1)}\left(k_{1}, k_{2}, p, S\right)\right], \\
& \tilde{W}_{\mu \nu}^{(2)}(q, p, S)=\frac{1}{2 \pi} \int \frac{\mathrm{d}^{4} k_{1}}{(2 \pi)^{4}} \frac{\mathrm{d}^{4} k_{2}}{(2 \pi)^{4}} \frac{\mathrm{d}^{4} k}{(2 \pi)^{4}} \sum_{c=L, R, M} \operatorname{Tr}\left[\hat{H}_{\mu \nu}^{(2, c) \rho \sigma}\left(x_{1}, x_{2}, x\right) \omega_{\rho}^{\rho^{\prime}} \omega_{\sigma}^{\sigma^{\prime}} \hat{\Phi}_{\rho^{\prime} \sigma^{\prime}}^{(2)}\left(k_{1}, k_{2}, k, p, S\right)\right],
\end{aligned}
$$

where $\hat{\Phi}^{(j)}$ 's are the gauge-invariant unintegrated quark-quark and quark-j-gluon(s)-quark correlators given by

$$
\begin{aligned}
& \hat{\Phi}^{(0)}(k, p, S)=\int \mathrm{d}^{4} y \mathrm{e}^{\mathrm{i} k y}\langle p, S|\bar{\psi}(0) \mathcal{L}(0 ; y) \psi(y)| p, S\rangle, \\
& \hat{\Phi}_{\rho}^{(1)}\left(k_{1}, k_{2}, p, S\right)=\int \mathrm{d}^{4} y \mathrm{~d}^{4} z \mathrm{e}^{\mathrm{i} k_{2} z+\mathrm{i} k_{1}(y-z)}\left\langle p, S\left|\bar{\psi}(0) \mathcal{L}(0 ; z) D_{\rho}(z) \mathcal{L}(z ; y) \psi(y)\right| p, S\right\rangle, \\
& \hat{\Phi}_{\rho \sigma}^{(2)}\left(k_{1}, k_{2}, k, p, S\right) \\
& \quad=\int \mathrm{d}^{4} y \mathrm{~d}^{4} y^{\prime} \mathrm{d}^{4} z \mathrm{e}^{\mathrm{i} k_{1} y+\mathrm{i} k\left(y^{\prime}-y\right)+\mathrm{i} k_{2}\left(z-y^{\prime}\right)}\left\langle p, S\left|\bar{\psi}(0) \mathcal{L}(0 ; y) D_{\rho}(y) \mathcal{L}\left(y ; y^{\prime}\right) D_{\sigma}\left(y^{\prime}\right) \mathcal{L}\left(y^{\prime} ; z\right) \psi(z)\right| p, S\right\rangle .
\end{aligned}
$$

$D(y)$ is the covariant derivative and is defined as $D_{\rho}(y)=-\mathrm{i} \partial_{\rho}+g A_{\rho}(y)$. The factor $\mathcal{L}(0 ; y)$ is obtained during the summation of different contributions with the same hard part and is given by

$$
\begin{aligned}
\mathcal{L}(0 ; y) & =\mathcal{L}^{\dagger}(\infty ; 0) \mathcal{L}(\infty ; y), \\
\mathcal{L}(\infty ; y) & =P \mathrm{e}^{-\mathrm{i} g \int_{y^{-}}^{\infty} \mathrm{d} \xi^{-} A^{+}\left(y^{+}, \xi^{-}, \vec{y}_{\perp}\right)} \\
& =1-\mathrm{i} g \int_{y^{-}}^{\infty} \mathrm{d} \xi^{-} A^{+}\left(y^{+}, \xi^{-}, \vec{y}_{\perp}\right)+(-\mathrm{i} g)^{2} \int_{y^{-}}^{\infty} \mathrm{d} \xi^{-} \int_{\xi^{-}}^{\infty} \mathrm{d} \eta^{-} A^{+}\left(y^{+}, \xi^{-}, \vec{y}_{\perp}\right) A^{+}\left(y^{+}, \eta^{-}, \vec{y}_{\perp}\right)+\cdots,
\end{aligned}
$$

where $P$ stands for the path-ordered integral. $\mathcal{L}(0 ; y)$ is nothing else but the well-known gauge link, which makes the quark-quark or quark-j-gluon(s)-quark correlator, and thus the PDFs defined using them, gauge invariant.

In this way, we have constructed a theoretical framework for systematically calculating the contributions to the hadronic tensor at the leading order (LO) in pQCD but at leading as well as higher twists. The results are given in terms of the gauge-invariant parton distribution and correlation functions (generally referred to as PDFs).

We emphasize the following two further points derived directly from these expressions.

First, we note that after collinear expansion, the hard parts contained in the expressions for $\tilde{W}_{\mu \nu}^{(j)}$ 's, such as those given by Eqs. (2.37)-(2.39), are only functions of the longitudinal component $x$. They are independent of the other components of the parton momentum $k$. We can integrate over these components of $k$ and simplify them to

$$
\tilde{W}_{\mu \nu}^{(0)}(q, p, S)=\frac{1}{2 \pi} \int p^{+} \mathrm{d} x \operatorname{Tr}\left[\hat{H}_{\mu \nu}^{(0)}(x) \hat{\Phi}^{(0)}(x, p, S)\right],
$$

$$
\begin{aligned}
& \tilde{W}_{\mu \nu}^{(1)}(q, p, S)=\frac{1}{2 \pi} \int p^{+} \mathrm{d} x_{1} p^{+} \mathrm{d} x_{2} \sum_{c=L, R} \operatorname{Tr}\left[\hat{H}_{\mu \nu}^{(1, c) \rho}\left(x_{1}, x_{2}\right) \omega_{\rho}^{\rho^{\prime}} \hat{\Phi}_{\rho^{\prime}}^{(1)}\left(x_{1}, x_{2}, p, S\right)\right], \\
& \tilde{W}_{\mu \nu}^{(2)}(q, p, S)=\frac{1}{2 \pi} \int p^{+} \mathrm{d} x_{1} p^{+} \mathrm{d} x_{2} p^{+} \mathrm{d} x \sum_{c=L, R, M} \operatorname{Tr}\left[\hat{H}_{\mu \nu}^{(2, c) \rho \sigma}\left(x_{1}, x_{2}, x\right) \omega_{\rho}^{\rho^{\prime}} \omega_{\sigma}^{\sigma^{\prime}} \hat{\Phi}_{\rho^{\prime} \sigma^{\prime}}^{(2)}\left(x_{1}, x_{2}, x, p, S\right)\right],
\end{aligned}
$$


where the matrix elements $\hat{\Phi}$ 's are given by

$$
\begin{aligned}
& \hat{\Phi}^{(0)}(x, p, S) \equiv \int \frac{\mathrm{d}^{4} k}{(2 \pi)^{4}} \delta\left(k^{+}-x p^{+}\right) \hat{\Phi}^{(0)}(k, p, S)=\int \frac{\mathrm{d} y^{-}}{2 \pi} \mathrm{e}^{\mathrm{i} x p^{+} y^{-}}\left\langle p, S\left|\bar{\psi}(0) \mathcal{L}\left(0 ; y^{-}\right) \psi\left(y^{-}\right)\right| p, S\right\rangle, \\
& \hat{\Phi}_{\rho}^{(1)}\left(x_{1}, x_{2}, p, S\right) \equiv \int \frac{\mathrm{d}^{4} k_{1}}{(2 \pi)^{4}} \frac{\mathrm{d}^{4} k_{2}}{(2 \pi)^{4}} \delta\left(k_{1}^{+}-x_{1} p^{+}\right) \delta\left(k_{2}^{+}-x_{2} p^{+}\right) \hat{\Phi}^{(1)}\left(k_{1}, k_{2}, p, S\right) \\
& =\int \frac{\mathrm{d} y^{-}}{2 \pi} \frac{\mathrm{d} z^{-}}{2 \pi} \mathrm{e}^{\mathrm{i} x_{2} p^{+} z^{-}+\mathrm{i} x_{1} p^{+}\left(y^{-}-z^{-}\right)}\left\langle p, S\left|\bar{\psi}(0) \mathcal{L}\left(0 ; z^{-}\right) D_{\rho}\left(z^{-}\right) \mathcal{L}\left(z^{-} ; y^{-}\right) \psi\left(y^{-}\right)\right| p, S\right\rangle, \\
& \hat{\Phi}_{\rho \sigma}^{(2)}\left(x_{1}, x_{2}, x, p, S\right) \equiv \int \frac{\mathrm{d}^{4} k_{1}}{(2 \pi)^{4}} \frac{\mathrm{d}^{4} k_{2}}{(2 \pi)^{4}} \frac{\mathrm{d}^{4} k}{(2 \pi)^{4}} \delta\left(k_{1}^{+}-x_{1} p^{+}\right) \delta\left(k_{2}^{+}-x_{2} p^{+}\right) \delta\left(k^{+}-x p^{+}\right) \hat{\Phi}^{(2)}\left(k_{1}, k_{2}, k, p, S\right) \\
& =\int \frac{\mathrm{d} y^{-}}{2 \pi} \frac{\mathrm{d} y^{\prime-}}{2 \pi} \frac{\mathrm{d} z^{-}}{2 \pi} \mathrm{e}^{\mathrm{i} x_{1} p^{+} y^{-}+\mathrm{i} x p^{+}\left(y^{\prime-}-y^{-}\right)+\mathrm{i} x_{2} p^{+}\left(z^{-}-y^{-}\right)}\langle p, S| \bar{\psi}(0) \mathcal{L}\left(0 ; y^{-}\right) D_{\rho}\left(y^{-}\right) \mathcal{L}\left(y^{-} ; y^{\prime-}\right) \\
& \quad \times D_{\sigma}\left(y^{\prime-}\right) \mathcal{L}\left(y^{\prime-} ; z^{-}\right) \psi\left(z^{-}\right)|p, S\rangle .
\end{aligned}
$$

From these expressions, we see explicitly that only $x_{i}$ dependences of the quark-quark and/or quark- $j$-gluon-quark correlators are involved. This means that only one-dimensional imaging of the nucleon is relevant in inclusive DIS.

Second, owing to the existence of the projection operator $\omega_{\rho}^{\rho^{\prime}}$, the hard parts can be further simplified a great deal. They are given by

$$
\begin{aligned}
& \hat{H}_{\mu \nu}^{(0)}(x)=\pi \hat{h}_{\mu \nu}^{(0)} \delta\left(x-x_{B}\right), \\
& \hat{H}_{\mu \nu}^{(1, L) \rho}\left(x_{1}, x_{2}\right) \omega_{\rho}^{\rho^{\prime}}=\frac{\pi}{2 q \cdot p} \hat{h}_{\mu \nu}^{(1) \rho} \omega_{\rho}^{\rho^{\prime}} \delta\left(x_{1}-x_{B}\right), \\
& \hat{H}_{\mu \nu}^{(2, L) \rho \sigma}\left(x_{1}, x_{2}, x\right) \omega_{\rho}^{\rho^{\prime}} \omega_{\sigma}^{\sigma^{\prime}}=\frac{2 \pi}{(2 q \cdot p)^{2}}\left[\bar{n}^{\rho} \hat{h}_{\mu \nu}^{(1) \sigma}+\frac{\hat{N}_{\mu \nu}^{(2) \rho \sigma}}{x_{2}-x_{B}-i \varepsilon}\right] \omega_{\rho}^{\rho^{\prime}} \omega_{\sigma}^{\sigma^{\prime}} \delta\left(x_{1}-x_{B}\right), \\
& \hat{H}_{\mu \nu}^{(2, M) \rho \sigma}\left(x_{1}, x_{2}, x\right) \omega_{\rho}^{\rho^{\prime}} \omega_{\sigma}^{\sigma^{\prime}}=\frac{2 \pi}{(2 q \cdot p)^{2}} \hat{h}_{\mu \nu}^{(2) \rho \sigma} \omega_{\rho}^{\rho^{\prime}} \omega_{\sigma}^{\sigma^{\prime}} \delta\left(x-x_{B}\right),
\end{aligned}
$$

where $\hat{h}_{\mu \nu}^{(0)}=\gamma_{\mu} \not h \gamma_{\nu} / p^{+}, \hat{h}_{\mu \nu}^{(1) \rho}=\gamma_{\mu} \not \gamma^{\rho} \not h \gamma_{\nu}, \hat{h}_{\mu \nu}^{(2) \rho \sigma}=p^{+} \gamma_{\mu} \not h \gamma^{\rho} \not h \gamma^{\sigma} \not h \gamma_{\nu} / 2$, and $\hat{N}_{\mu \nu}^{(2) \rho \sigma}=q^{-} \gamma_{\mu} \gamma^{\rho} \not h \gamma^{\sigma} \gamma_{\nu}$ are matrices independent of $x_{i}$. We insert them into Eqs. (2.45)-(2.47) and obtain the simplified expressions for the hadronic tensor as

$$
\begin{aligned}
& \tilde{W}_{\mu \nu}^{(0)}(q, p, S)=\frac{1}{2} \operatorname{Tr}\left[\hat{h}_{\mu \nu}^{(0)} \hat{\Phi}^{(0)}\left(x_{B}\right)\right] \\
& \tilde{W}_{\mu \nu}^{(1, L)}(q, p, S)=\frac{1}{4 q \cdot p} \operatorname{Tr}\left[\hat{h}_{\mu \nu}^{(1) \rho} \omega_{\rho}^{\rho^{\prime}} \hat{\varphi}_{\rho^{\prime}}^{(1)}\left(x_{B}\right)\right] \\
& \tilde{W}_{\mu \nu}^{(2, L)}(q, p, S)=\frac{1}{(2 q \cdot p)^{2}}\left\{\operatorname{Tr}\left[\hat{h}_{\mu \nu}^{(1) \rho} \omega_{\rho}^{\rho^{\prime}} \hat{\phi}_{\rho^{\prime}}^{(2 L)}\left(x_{B}\right)\right]+\operatorname{Tr}\left[\hat{N}_{\mu \nu}^{(2) \rho \sigma} \omega_{\rho}^{\rho^{\prime}} \omega_{\sigma}^{\sigma^{\prime}} \hat{\varphi}_{\rho^{\prime} \sigma^{\prime}}^{(2 L)}\left(x_{B}\right)\right]\right\}, \\
& \tilde{W}_{\mu \nu}^{(2, M)}(q, p, S)=\frac{1}{(2 q \cdot p)^{2}} \operatorname{Tr}\left[\hat{h}_{\mu \nu}^{(2) \rho \sigma} \omega_{\rho}^{\rho^{\prime}} \omega_{\sigma^{\sigma^{\prime}}}^{\sigma^{\prime}} \hat{\varphi}_{\rho^{\prime} \sigma^{\prime}}^{(2 M)}\left(x_{B}\right)\right],
\end{aligned}
$$

where, for explicitness, we omit $p, S$ in the arguments of the correlators. These correlators are defined as

$$
\begin{aligned}
& \hat{\varphi}_{\rho}^{(1)}\left(x_{1}\right) \equiv \int \mathrm{d} x_{2} \hat{\Phi}_{\rho}^{(1)}\left(x_{1}, x_{2}, p, S\right)=\int \frac{p^{+} \mathrm{d} y^{-}}{2 \pi} \mathrm{e}^{\mathrm{i} x p^{+} y^{-}}\left\langle p, S\left|\bar{\psi}(0) D_{\rho}(0) \mathcal{L}\left(0 ; y^{-}\right) \psi\left(y^{-}\right)\right| p, S\right\rangle, \\
& \hat{\varphi}_{\rho \sigma}^{(2 M)}(x) \equiv \int \mathrm{d} x_{1} \mathrm{~d} x_{2} \hat{\Phi}_{\rho \sigma}^{(2)}\left(x_{1}, x_{2}, x, p, S\right)=\int \frac{p^{+} \mathrm{d} y^{-}}{2 \pi} \mathrm{e}^{\mathrm{i} x p^{+} y^{-}}\left\langle p, S\left|\bar{\psi}(0) D_{\rho}(0) \mathcal{L}\left(0 ; y^{-}\right) D_{\sigma}\left(y^{-}\right) \psi\left(y^{-}\right)\right| p, S\right\rangle, \\
& \hat{\varphi}_{\rho \sigma}^{(2 L)}\left(x_{1}\right) \equiv \int \frac{\mathrm{d} x \mathrm{~d} x_{2}}{x_{2}-x_{1}-\mathrm{i} \varepsilon} \hat{\Phi}_{\rho \sigma}^{(2)}\left(x_{1}, x_{2}, x, p, S\right) \\
& =\int \frac{\mathrm{d} x_{2}}{x_{2}-x-\mathrm{i} \epsilon} \frac{p^{+} \mathrm{d} y^{-}}{2 \pi} \frac{p^{+} \mathrm{d} z^{-}}{2 \pi} \mathrm{e}^{\mathrm{i} x p^{+} y^{-}+\mathrm{i}\left(x_{2}-x\right) p^{+} z^{-}}\left\langle p, S\left|\bar{\psi}(0) \mathcal{L}\left(0 ; z^{-}\right) D_{\rho}\left(z^{-}\right) D_{\sigma}\left(z^{-}\right) \mathcal{L}\left(z^{-} ; y^{-}\right) \psi\left(y^{-}\right)\right| p, S\right\rangle, \\
& \hat{\phi}_{\sigma}^{(2 L)}\left(x_{1}\right) \equiv \int \mathrm{d} x \mathrm{~d} x_{2} \bar{n}^{\rho} \hat{\Phi}_{\rho \sigma}^{(2)}\left(x_{1}, x_{2}, x, p, S\right)=\int \frac{p^{+} \mathrm{d} y^{-}}{2 \pi} \mathrm{e}^{\mathrm{i} x p^{+} y^{-}}\left\langle p, S\left|\bar{\psi}(0) D^{-}(0) D_{\sigma}(0) \mathcal{L}\left(0 ; y^{-}\right) \psi\left(y^{-}\right)\right| p, S\right\rangle .
\end{aligned}
$$


We see explicitly that all the relevant components of the quark- $j$-gluon-quark correlators depend only on one single parton momentum. This means that only quark $-j$ gluon-quark correlators that depend on one single parton momentum are relevant in inclusive DIS.

We emphasize that the results given by Eqs. (2.37)(2.39) and their simplified forms given by Eqs. (2.55)(2.62), including the gauge links, are derived in the collinear expansion. They are just the sum of the contributions from the diagram series shown in Fig. 1. This formalism provides a basic theoretical framework for describing inclusive DIS at LO pQCD but at leading and higher twist contributions in terms of gauge-invariant PDFs.

The PDFs are defined in terms of QFT operators via these quark-quark correlators by expanding them in terms of $\gamma$ matrices and basic Lorentz covariants. For example, for $\hat{\Phi}^{(0)}(x, p, S)$, we have

$$
\begin{gathered}
\hat{\Phi}^{(0)}(x)=\frac{1}{2}\left[\Phi_{S}^{(0)}(x)+\mathrm{i} \gamma_{5} \Phi_{P S}^{(0)}(x)+\gamma^{\alpha} \Phi_{\alpha}^{(0)}(x)\right. \\
\left.+\gamma_{5} \gamma^{\alpha} \tilde{\Phi}_{\alpha}^{(0)}(x)+\mathrm{i} \sigma^{\alpha \beta} \gamma_{5} \Phi_{T \alpha \beta}^{(0)}(x)\right] .
\end{gathered}
$$

The basic Lorentz covariants are constructed from $p_{\alpha}$, $n_{\alpha}, S_{\alpha}$, and $\varepsilon_{\alpha \beta \rho \sigma}$. We obtain the following general results:

$$
\begin{aligned}
& \Phi_{S}^{(0)}(x)=M e(x) \\
& \Phi_{P S}^{(0)}(x)=\lambda M e_{L}(x) \\
& \Phi_{\alpha}^{(0)}(x)=p^{+} \bar{n}_{\alpha} f_{1}(x)+M \varepsilon_{\perp \alpha \rho} S_{T}^{\rho} f_{T}(x)+\frac{M^{2}}{p^{+}} n_{\alpha} f_{3}(x)
\end{aligned}
$$

$$
\begin{aligned}
\tilde{\Phi}_{\alpha}^{(0)}(x)= & \lambda p^{+} \bar{n}_{\alpha} g_{1 L}(x)+M S_{T \alpha} g_{T}(x) \\
& +\lambda \frac{M^{2}}{p^{+}} n_{\alpha} g_{3 L}(x),
\end{aligned}
$$$$
\Phi_{T \rho \alpha}^{(0)}(x)=p^{+} \bar{n}_{[\rho} S_{T \alpha]} h_{1 T}(x)-M \varepsilon_{\perp \rho \alpha} h(x)
$$$$
+\lambda M \bar{n}_{[\rho} n_{\alpha]} h_{L}(x)+\frac{M^{2}}{p^{+}} n_{[\rho} S_{T \alpha]} h_{3 T}(x),
$$

where $\varepsilon_{\perp \rho \sigma} \equiv \varepsilon_{\alpha \beta \rho \sigma} \bar{n}^{\alpha} n^{\beta}$, and the anticommutation symbol $A^{[\rho} B^{\sigma]} \equiv A^{\rho} B^{\sigma}-A^{\sigma} B^{\rho}$. The scalar functions $f(x), g(x)$, and $h(x)$ are the corresponding PDFs. There are a total of 12 such functions; 3 of them, i.e., $f_{1}(x)$, $g_{1 L}(x)$, and $h_{1 T}(x)$, contribute at leading twist and have clear probability interpretations, whereas 6 of them contribute at twist- 3 , and the other 3 contribute at twist4. We further note that in fact the three time-reversal odd terms $e_{L}(x), f_{T}(x)$, and $h(x)$ vanish in the onedimensional case. We keep them in Eqs. (2.65)-(2.68) for later comparison with fragmentation functions.

We also see that the PDFs involved here are all scale independent. This is because we have so far considered only the LO pQCD contributions, i.e., the tree diagrams. To go to higher orders of pQCD, we take the loop diagrams, gluon radiation, and so on into account. After proper handling of these contributions, we obtain the factorized form [6], in which the PDFs acquire the scale $(Q)$ dependence governed by the QCD evolution equations. In practice, $\mathrm{PDF}$ are parameterized and are given in the PDF library (PDFlib).

In summary, to study one-dimensional imaging of the nucleon with inclusive DIS, we take the following steps.

- General symmetry analysis leads to the general form of the hadronic tensor and/or the cross section in terms of four independent structure functions.

- The parton model without QCD interaction leads to LO in pQCD and leading twist results for the structure functions in terms of $Q$-independent PDFs without (local) gauge invariance.

- The parton model with QCD multiple gluon scattering after collinear expansion leads to LO in pQCD and leading and higher twist contributions in terms of $Q$-independent but gauge-invariant PDFs.

- The parton model with QCD multiple gluon scattering and loop diagram contributions after collinear approximation, regularization, and renormalization leads to leading and higher-order pQCD and leading twist contributions in factorized forms in terms of $Q$-evolved and gauge-invariant PDFs.

In the following, we will follow these four steps and summarize what we have achieved in the threedimensional case. As in Ref. [2], we will focus mainly on the theoretical framework at LO pQCD but consistently take leading and higher twist contributions into account. Before that, we emphasize the following two historical developments that may be helpful in constructing the theoretical framework for the TMD case.

First, as mentioned, the study of three-dimensional imaging of the nucleon was triggered by the experimental observation of single-spin left-right asymmetries (SSAs) in the inclusive hadron-hadron collision with a transversely polarized projectile or target. It was known that pQCD leads to negligibly small asymmetry for the hard part [12], but the observed asymmetry can be as large as $40 \%$ [13]. The hunt for such large asymmetries has lasted for decades, with the following milestones:

- In 1991, Sivers introduced [14] the asymmetric quark distribution in a transversely polarized nucleon, which is now known as the Sivers function. 
- In 1993, Boros, Liang, and Meng proposed [15] a phenomenological model that provides an intuitive physical picture showing that the asymmetry arises from the orbital angular momenta of quarks and what they called the surface effect caused by the initial- or final-state interactions.

- In 1993, Collins published [16] his proof that the Sivers function has to vanish because of parity and time-reversal invariance.

- In 2002, Brodsky, Hwang, and Schmidt calculated [17] the SSA for semi-inclusive deep inelastic scattering (SIDIS) using an explicit example in which they took the orbital angular momentum of quarks and multiple gluon scattering into account.

- In 2002, immediately after [17], Collins pointed out [18] that multiple gluon scattering is contained in the gauge link and that the conclusion of his proof in 1993 was incorrect because he did not consider the gauge link. He further showed that by taking the gauge link into account, the same proof leads to the conclusion that the Sivers function for DIS and that for the Drell-Yan process have opposite signs. Belitsky, Ji, and Yuan resolved [19, 20] the problem of defining the gauge link for a TMD parton density in the light-cone gauge where the gauge potential does not vanish asymptotically.

The second historical development concerns the study of azimuthal asymmetry in SIDIS. Georgi and Politzer showed in 1977 [21] that final-state gluon radiation leads to azimuthal asymmetries and could be used as a "clean test" of pQCD. However, soon after, in 1978, Cahn showed [22] that similar asymmetries can also be obtained if one includes the intrinsic transverse momenta of partons. The latter (now called the Cahn effect), although power suppressed at higher twist, can be quite significant and cannot be neglected, as the values of the asymmetries themselves are usually not very large.

The following two points are particularly valuable lessons that we learned from these historical developments: when studying TMDs,

- it is important to take the gauge link into account; - higher twist effects can be important.

Both of these points demand that, to describe SIDIS in terms of TMDs, we need the proper QFT formulation rather than the intuitive parton model.

\section{TMDs defined via quark-quark correlator}

The TMD PDFs of quarks are defined via the TMD quark-quark correlator $\Phi^{(0)}\left(x, k_{\perp} ; p, S\right)$ given by Eq. (2.40) (after integration over $k^{-}$). A systematical study is given in Ref. [23], and a very comprehensive treatment can also be found in Ref. [24]. Here, we first expand it in terms of $\gamma$ matrices and obtain a scalar, a pseudoscalar, a vector, an axial vector, and an antisymmetric and spacereflection odd tensor part, i.e.,

$$
\begin{aligned}
\hat{\Phi}^{(0)}\left(x, k_{\perp} ; p, S\right)= & \frac{1}{2}\left[\Phi_{S}^{(0)}\left(x, k_{\perp} ; p, S\right)+\mathrm{i} \gamma_{5} \Phi_{P S}^{(0)}\left(x, k_{\perp} ; p, S\right)+\gamma^{\alpha} \Phi_{\alpha}^{(0)}\left(x, k_{\perp} ; p, S\right)\right. \\
& \left.+\gamma_{5} \gamma^{\alpha} \tilde{\Phi}_{\alpha}^{(0)}\left(x, k_{\perp} ; p, S\right)+\mathrm{i} \sigma^{\alpha \beta} \gamma_{5} \Phi_{T \alpha \beta}^{(0)}\left(x, k_{\perp} ; p, S\right)\right] .
\end{aligned}
$$

The operator expressions of these coefficients are given by the traces of the quark-quark correlator with the corresponding Dirac matrices. For example, for the vector component, we have

$$
\Phi_{\alpha}^{(0)}\left(x, k_{\perp} ; p, S\right)=\frac{1}{2} \operatorname{Tr}\left[\gamma_{\alpha} \hat{\Phi}^{(0)}\left(x, k_{\perp} ; p, S\right)\right]=\int \mathrm{d} z^{-} \mathrm{d}^{2} z_{\perp} \mathrm{e}^{\mathrm{i}\left(x p^{+} z^{-}-\vec{k}_{\perp} \cdot \vec{z}_{\perp}\right)}\left\langle p, S\left|\bar{\psi}(0) \mathcal{L}(0 ; z) \frac{\gamma_{\alpha}}{2} \psi(z)\right| p, S\right\rangle .
$$

We then analyze the Lorentz structure of each part by expressing it in terms of possible "basic Lorentz covariants" and scalar functions. From $\hat{\Phi}^{(0)}\left(x, k_{\perp} ; p, S\right)$, we obtain the results as [23]

$$
\begin{aligned}
\Phi_{S}^{(0)}\left(x, k_{\perp} ; p, S\right)= & M\left[e\left(x, k_{\perp}\right)-\frac{\varepsilon_{\perp \rho \sigma} k_{\perp}^{\rho} S_{T}^{\sigma}}{M} e_{T}^{\perp}\left(x, k_{\perp}\right)\right], \\
\Phi_{P S}^{(0)}\left(x, k_{\perp} ; p, S\right)= & M\left[\lambda e_{L}\left(x, k_{\perp}\right)-\frac{k_{\perp} \cdot S_{T}}{M} e_{T}\left(x, k_{\perp}\right)\right], \\
\Phi_{\alpha}^{(0)}\left(x, k_{\perp} ; p, S\right)= & p^{+} \bar{n}_{\alpha}\left[f_{1}\left(x, k_{\perp}\right)-\frac{\varepsilon_{\perp \rho \sigma} k_{\perp}^{\rho} S_{T}^{\sigma}}{M} f_{1 T}^{\perp}\left(x, k_{\perp}\right)\right]+k_{\perp \alpha}\left[f^{\perp}\left(x, k_{\perp}\right)-\frac{\varepsilon_{\perp \rho \sigma} k_{\perp}^{\rho} S_{T}^{\sigma}}{M} f_{T}^{\perp 1}\left(x, k_{\perp}\right)\right] \\
& +\varepsilon_{\perp \alpha \rho} k_{\perp}^{\rho}\left[\lambda f_{L}^{\perp}\left(x, k_{\perp}\right)-\frac{k_{\perp} \cdot S_{T}}{M} f_{T}^{\perp 2}\left(x, k_{\perp}\right)\right]+\frac{M^{2}}{p^{+}} n_{\alpha}\left[f_{3}\left(x, k_{\perp}\right)-\frac{\varepsilon_{\perp \rho \sigma} k_{\perp}^{\rho} S_{T}^{\sigma}}{M} f_{3 T}^{\perp}\left(x, k_{\perp}\right)\right], \\
\tilde{\Phi}_{\alpha}^{(0)}\left(x, k_{\perp} ; p, S\right)= & p^{+} \bar{n}_{\alpha}\left[\lambda g_{1 L}\left(x, k_{\perp}\right)-\frac{k_{\perp} \cdot S_{T}}{M} g_{1 T}^{\perp}\left(x, k_{\perp}\right)\right]+M S_{T \alpha} g_{T}^{\prime}\left(x, k_{\perp}\right)-\varepsilon_{\perp \alpha \beta} k_{\perp}^{\beta} g^{\perp}\left(x, k_{\perp}\right)
\end{aligned}
$$




$$
\begin{aligned}
& \quad+k_{\perp \alpha}\left[\lambda g_{L}^{\perp}\left(x, k_{\perp}\right)-\frac{k_{\perp} \cdot S_{T}}{M} g_{T}^{\perp}\left(x, k_{\perp}\right)\right]+\frac{M^{2}}{p^{+}} n_{\alpha}\left[\lambda g_{3 L}\left(x, k_{\perp}\right)-\frac{k_{\perp} \cdot S_{T}}{M} g_{3 T}\left(x, k_{\perp}\right)\right] \\
& \Phi_{T \rho \alpha}^{(0)}\left(x, k_{\perp} ; p, S\right)=p^{+} \bar{n}_{[\rho} S_{T \alpha]} h_{1 T}\left(x, k_{\perp}\right)-\frac{p^{+} \bar{n}_{[\rho} \varepsilon_{\perp \alpha] \beta} k_{\perp}^{\beta}}{M} h_{1}^{\perp}\left(x, k_{\perp}\right)+\frac{p^{+} \bar{n}_{[\rho} k_{\perp \alpha]}}{M}\left[\lambda h_{1 L}^{\perp}\left(x, k_{\perp}\right)-\frac{k_{\perp} \cdot S_{T}}{M} h_{1 T}^{\perp}\left(x, k_{\perp}\right)\right] \\
& +S_{T[\rho} k_{\perp \alpha]} h_{T}^{\perp}\left(x, k_{\perp}\right)-M \varepsilon_{\perp \rho \alpha} h\left(x, k_{\perp}\right)+M \bar{n}_{[\rho} n_{\alpha]}\left[\lambda h_{L}\left(x, k_{\perp}\right)-\frac{k_{\perp} \cdot S_{T}}{M} h_{T}\left(x, k_{\perp}\right)\right] \\
& \quad+\frac{M^{2}}{p^{+}}\left\{n_{[\rho} S_{T \alpha]} h_{3 T}\left(x, k_{\perp}\right)+\frac{n_{[\rho} k_{\perp \alpha]}}{M}\left[\lambda h_{3 L}^{\perp}\left(x, k_{\perp}\right)-\frac{k_{\perp} \cdot S_{T}}{M} h_{3 T}^{\perp}\left(x, k_{\perp}\right)\right]-\frac{n_{[\rho} \varepsilon_{\perp \alpha] \beta} k_{\perp}^{\beta}}{M} h_{3}^{\perp}\left(x, k_{\perp}\right)\right\}
\end{aligned}
$$

These scalar functions are known as TMD PDFs. There are a total of 32 such TMD PDFs. Among them, 8 contribute at leading twist, and they all have clear probability interpretations such as the number density $f_{1}\left(x, k_{\perp}\right)$, helicity distribution $g_{1 L}\left(x, k_{\perp}\right)$, transversity $h_{1 T}\left(x, k_{\perp}\right)$, Sivers function $f_{1 T}^{\perp}\left(x, k_{\perp}\right)$, and Boer-Mulders function $h_{1}^{\perp}\left(x, k_{\perp}\right) ; 16$ contribute at twist- 3 , and the other 8 contribute at twist- 4 . We emphasize that they are all scalar functions of $x$ and $k_{\perp}$; i.e., they depend on $x$ and $k_{\perp}^{2}$.

If we integrate over $d^{2} k_{\perp}$, terms in which the basic Lorentz covariants are odd in $k_{\perp}$ vanish. Eqs. (3.3)-(3.7) just reduce to the corresponding Eqs. (2.64)-(2.68). At the leading twist, only 3 of the 8 survive: the number density $f_{1}(x)$, helicity distribution $g_{1 L}(x)$, and transversity $h_{1 T}(x)$.

We show the leading twist and twist-3 TMD PDFs in Tables 1 and 2, respectively. In these tables, we show also the results for $\mathcal{L}=1$, i.e., if we neglect multiple gluon scattering and simply take a nucleon as an ideal gas system consisting of quarks and antiquarks (see, e.g., $[24])$. We also note that the conventions used here have the following systematics: $f, g$, and $h$ represent unpolarized, longitudinally polarized, and transversely polarized quarks, respectively; the subscript $L$ or $T$ stands for longitudinally or transversely polarized nucleons, respectively; the subscript 1 indicates leading twist, no number indicates twist- 3 , and subscript 3 indicates twist-4; the symbol $\perp$ in the superscript denotes that the correspond- ing basic Lorentz covariant is $k_{\perp}$ dependent.

Higher twist TMD PDFs are also defined via quark$j$-gluon(s)-quark correlators such as those given by Eqs. (2.59)-(2.62). Many of them, however, are not independent, as they are related to those defined via the quarkquark correlator through the QCD equation of motion, $\gamma \cdot D(z) \psi(z)=0$. We can obtain relations such as

$$
\begin{aligned}
x \Phi_{\perp \rho}^{(0)}\left(x, k_{\perp} ; p, S\right)= & -\frac{n^{\alpha}}{p^{+}}\left[\operatorname{Re} \varphi_{\alpha \rho}^{(1)}\left(x, k_{\perp} ; p, S\right)\right. \\
& \left.+\varepsilon_{\perp \rho}{ }^{\sigma} \operatorname{Im} \tilde{\varphi}_{\alpha \sigma}^{(1)}\left(x, k_{\perp} ; p, S\right)\right], \\
x \tilde{\Phi}_{\perp \rho}^{(0)}\left(x, k_{\perp} ; p, S\right)= & -\frac{n^{\alpha}}{p^{+}}\left[\operatorname{Re} \tilde{\varphi}_{\alpha \rho}^{(1)}\left(x, k_{\perp} ; p, S\right)\right. \\
& \left.+\varepsilon_{\perp \rho}{ }^{\sigma} \operatorname{Im} \varphi_{\alpha \sigma}^{(1)}\left(x, k_{\perp} ; p, S\right)\right] .
\end{aligned}
$$

It is interesting to see that [35], although it is not generally proved, all the twist-3 TMD PDFs that are defined via the quark-gluon-quark correlator $\varphi_{\rho}^{(1)}$ and involved in SIDIS are replaced by those defined via the quarkquark correlator $\Phi^{(0)}$.

We emphasize that fragmentation is just conjugate to parton distribution. A systematic study of the general structure of the fragmentation function (FF) defined via

\begin{tabular}{|c|c|c|c|c|c|}
\hline $\begin{array}{c}\text { Quark } \\
\text { polarization } \\
\end{array}$ & $\begin{array}{c}\text { Nucleon } \\
\text { polarization }\end{array}$ & TMD PDFs & if $\mathcal{L}=1$ & integrated over $\vec{k}_{\perp}$ & Name \\
\hline$U$ & $U$ & $f_{1}\left(x, k_{\perp}\right)$ & & $f_{1}(x)$ & Number density \\
\hline \multirow{2}{*}{$L$} & $L$ & $g_{1 L}\left(x, k_{\perp}\right)$ & & $g_{1 L}(x)$ & Helicity distribution \\
\hline & $T$ & $g_{1 T}^{\perp}\left(x, k_{\perp}\right)$ & & $\times$ & Worm-gear/Trans-helicity distribution \\
\hline \multirow{3}{*}{$T$} & $\bar{U}$ & $h_{1}^{\perp}\left(x, k_{\perp}\right)$ & 0 & $\bar{x}$ & Boer-Mulders function \\
\hline & $T(\perp)$ & $h_{1 T}^{\perp}\left(x, k_{\perp}\right)$ & & & Pretzelosity \\
\hline & $L$ & $h_{1 L}^{\perp}\left(x, k_{\perp}\right)$ & & $\times$ & Worm-gear/longi-transversity \\
\hline
\end{tabular}
the corresponding quark-quark correlator is presented in Ref. [26]. We should have one-to-one correspondence between TMD PDFs and TMD FFs. E.g., corresponding to the quark-quark correlator $\Phi^{(0)}(k, p, S)$ given by Eq. (2.40) and the expanded form in Eq. (3.1), we have

Table 1 The 8 leading twist TMD PDFs defined via the quark-quark correlator. A $\times$ means that the corresponding term disappears upon integrating the quark-quark correlator over $d^{2} k_{\perp}$. 
Table 2 The 16 twist-3 TMD PDFs defined via the quark-quark correlator. A $\times$ means that the corresponding term disappears upon integrating the quark-quark correlator over $\mathrm{d}^{2} k_{\perp}$.

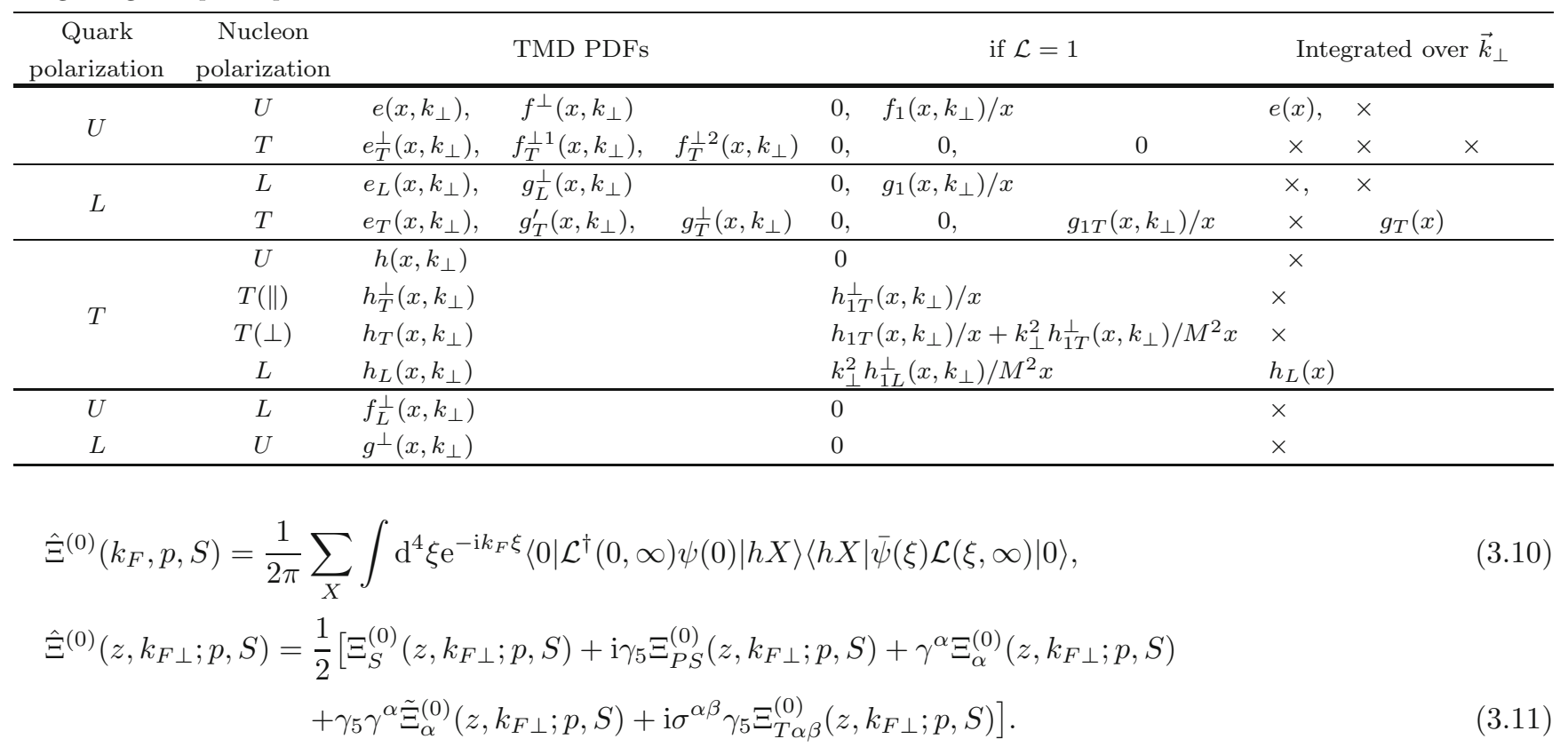

For a spin-1/2 hadron, we have perfect one-to-one correspondence to those given by Eqs. (3.3)-(3.7) for parton distributions in the nucleon, i.e.,

$$
\begin{aligned}
& z \Xi_{S}^{(0)}\left(z, k_{F \perp} ; p, S\right)=M\left[E\left(z, k_{F \perp}\right)+\frac{\varepsilon_{\perp \rho \sigma} k_{F \perp}^{\rho} S_{T}^{\sigma}}{M} E_{T}^{\perp}\left(z, k_{F \perp}\right)\right], \\
& z \Xi_{P S}^{(0)}\left(z, k_{F \perp} ; p, S\right)=M\left[\lambda E_{L}\left(z, k_{F \perp}\right)+\frac{k_{F \perp} \cdot S_{T}}{M} E_{T}\left(z, k_{F \perp}\right)\right], \\
& z \Xi_{\alpha}^{(0)}\left(z, k_{F \perp} ; p, S\right)=p^{+} \bar{n}_{\alpha}\left[D_{1}\left(z, k_{F \perp}\right)+\frac{\varepsilon_{\perp \rho \sigma} k_{F \perp}^{\rho} S_{T}^{\sigma}}{M} D_{1 T}^{\perp}\left(z, k_{F \perp}\right)\right]+k_{F \perp \alpha} D^{\perp}\left(z, k_{F \perp}\right)+M \varepsilon_{\perp \alpha \rho} S_{T}^{\rho} D_{T}\left(z, k_{F \perp}\right), \\
& \quad+\varepsilon_{\perp \alpha \rho} k_{F \perp}^{\rho}\left[\lambda D_{L}^{\perp}\left(z, k_{F \perp}\right)+\frac{k_{F \perp} \cdot S_{T}}{M} D_{T}^{\perp}\left(z, k_{F \perp}\right)\right]+\frac{M^{2}}{p^{+}} n_{\alpha}\left[D_{3}\left(z, k_{F \perp}\right)+\frac{\varepsilon_{\perp \rho \sigma} k_{F \perp}^{\rho} S_{T}^{\sigma}}{M} D_{3 T}^{\perp}\left(z, k_{F \perp}\right)\right], \quad(3.14) \\
& z \tilde{\Xi}_{\alpha}^{(0)}\left(z, k_{F \perp} ; p, S\right)=p^{+} \bar{n}_{\alpha}\left[\lambda G_{1 L}\left(z, k_{F \perp}\right)+\frac{k_{F \perp} \cdot S_{T}}{M} G_{1 T}^{\perp}\left(z, k_{F \perp}\right)\right]+M S_{T \alpha} G_{T}\left(z, k_{F \perp}\right)+\varepsilon_{\perp \alpha \beta} k_{F \perp}^{\beta} G^{\perp}\left(z, k_{F \perp}\right) \\
& \quad+k_{F \perp \alpha}\left[\lambda G_{L}^{\perp}\left(z, k_{F \perp}\right)+\frac{k_{F \perp} \cdot S_{T}}{M} G_{T}^{\perp}\left(z, k_{F \perp}\right)\right]+\frac{M^{2}}{p^{+}} n_{\alpha}\left[\lambda G_{3 L}\left(z, k_{F \perp}\right)+\frac{k_{F \perp} \cdot S_{T}}{M} G_{3 T}\left(z, k_{F \perp}\right)\right], \\
& z \Xi_{T \rho \alpha}^{(0)}\left(z, k_{F \perp} ; p, S\right)=p^{+} \bar{n}_{[\rho} S_{T \alpha]} H_{1 T}\left(z, k_{F \perp}\right)+\frac{p^{+} \bar{n}_{[\rho} \varepsilon_{\perp \alpha] \beta} k_{F \perp}^{\beta}}{M} H_{1}^{\perp}\left(z, k_{F \perp}\right) \\
& \quad+\frac{p^{+} \bar{n}_{[\rho} k_{F \perp \alpha]}}{M}\left[\lambda H_{1 L}^{\perp}\left(z, k_{F \perp}\right)+\frac{k_{F \perp} \cdot S_{T}}{M} H_{1 T}^{\perp}\left(z, k_{F \perp}\right)\right]+S_{T[\rho} k_{F \perp \alpha]} H_{T}^{\perp}\left(z, k_{F \perp}\right)+M \varepsilon_{\perp \rho \alpha} H\left(z, k_{F \perp}\right) \\
& \quad+\bar{n}_{[\rho} n_{\alpha]}\left[M \lambda H_{L}\left(z, k_{F \perp}\right)+k_{F \perp} \cdot S_{T} H_{T}^{\perp \perp}\left(z, k_{F \perp}\right)\right]+\frac{M^{2}}{p^{+}}\left\{n_{[\rho} S_{T \alpha]} H_{3 T}\left(z, k_{F \perp}\right)+\frac{n_{[\rho} \varepsilon_{\perp \alpha] \beta} k_{F \perp}^{\beta}}{M} H_{3}^{\perp}\left(z, k_{F \perp}\right)\right. \\
& \left.\quad+\frac{n_{[\rho} k_{F \perp \alpha]}}{M}\left[\lambda H_{3 L}^{\perp}\left(z, k_{F \perp}\right)+\frac{k_{F \perp} \cdot S_{T}}{M} H_{3 T}^{\perp}\left(z, k_{F \perp}\right)\right]\right\} .
\end{aligned}
$$

Comparing them with the results given by Eqs. (3.3)(3.7), we see clearly the one-to-one correspondence between the FFs and PDFs. As an example, we show the eight leading twist components in Table 3 . We do not show the results for $\mathcal{L}=1$ for FFs. This is because even if we neglect the multiple gluon scattering that leads to the gauge link, final-state interactions can still exist between $h$ and $X$. In this case, time-reversal invariance does not lead to zero results for the T-odd amplitudes.

For spin- 1 hadrons, the polarization is described by 
the polarization vector $S$ and also the polarization tensor $T$ (see, e.g., [25] and [26]). The tensor polarization part has five independent components. They are given by a Lorentz scalar $S_{L L}$, a Lorentz vector $S_{L T}^{\mu}=\left(0, S_{L T}^{x}, S_{L T}^{y}, 0\right)$, and a Lorentz tensor $S_{T T}^{\mu \nu}$, which has two independent nonzero components, $S_{T T}^{x x}$ and $S_{T T}^{x y}$, in the rest frame of the hadron. These polarization parameters can be related to the probabilities for particles in different spin states [25]. In this case, the TMD quark-quark correlator $\hat{\Xi}^{(0)}\left(z, k_{F \perp} ; p, S\right)$ is decomposed into a spin-independent part, a vector-polarizationdependent part, and a tensor-polarization-dependent part; i.e., $\hat{\Xi}^{(0)}\left(z, k_{F \perp} ; p, S\right)=\hat{\Xi}^{U(0)}\left(z, k_{F \perp} ; p, S\right)+$ $\hat{\Xi}^{V(0)}\left(z, k_{F \perp} ; p, S\right)+\hat{\Xi}^{T(0)}\left(z, k_{F \perp} ; p, S\right)$. The spinindependent and vector-polarization-dependent part $\hat{\Xi}^{U+V(0)}\left(z, k_{F \perp} ; p, S\right)$ takes exactly the same decomposition as that for the spin- $1 / 2$ hadron given by Eqs. (3.12)-(3.16). The tensor-polarization-dependent part is presented in Ref. [26] and is given by

Table 3 The 8 leading twist TMD FFs for spin- $1 / 2$ hadrons defined via the quark-quark correlator. A $\times$ means that the corresponding term disappears upon integrating the quark-quark correlator over $\mathrm{d}^{2} k_{F \perp}$.

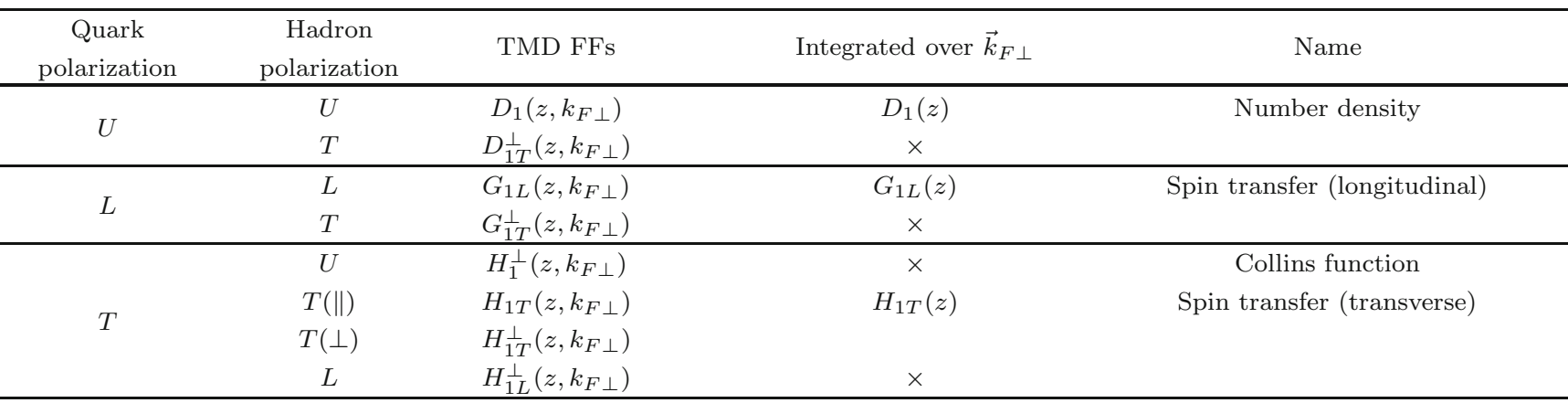

$$
\begin{aligned}
& z \Xi_{S}^{T(0)}\left(z, k_{F \perp} ; p, S\right)=M\left[S_{L L} E_{L L}\left(z, k_{F \perp}\right)+\frac{k_{F \perp} \cdot S_{L T}}{M} E_{L T}^{\perp}\left(z, k_{F \perp}\right)+\frac{k_{F \perp} \cdot S_{T T} \cdot k_{F \perp}}{M^{2}} E_{T T}^{\perp}\left(z, k_{F \perp}\right)\right] \\
& z \Xi_{P S}^{T(0)}\left(z, k_{F \perp} ; p, S\right)=M\left[\frac{\epsilon_{\perp}^{k_{F} S_{L T}}}{M} E_{L T}^{\perp}\left(z, k_{F \perp}\right)+\frac{\epsilon_{\perp k_{F} \alpha} k_{\beta} S_{T T}^{\alpha \beta}}{M^{2}} E_{T T}^{\prime \perp}\left(z, k_{F \perp}\right)\right] \\
& z \Xi_{\alpha}^{T(0)}\left(z, k_{F \perp} ; p, S\right)=p^{+} \bar{n}_{\alpha}\left[S_{L L} D_{1 L L}\left(z, k_{F \perp}\right)+\frac{k_{F \perp} \cdot S_{L T}}{M} D_{1 L T}^{\perp}\left(z, k_{F \perp}\right)+\frac{k_{F \perp} \cdot S_{T T} \cdot k_{F \perp}}{M^{2}} D_{1 T T}^{\perp}\left(z, k_{F \perp}\right)\right] \\
& +k_{F \perp \alpha}\left[S_{L L} D_{L L}\left(z, k_{F \perp}\right)+\frac{k_{F \perp} \cdot S_{L T}}{M} D_{L T}^{\perp}\left(z, k_{F \perp}\right)+\frac{k_{F \perp} \cdot S_{T T} \cdot k_{F \perp}}{M^{2}} D_{T T}^{\perp}\left(z, k_{F \perp}\right)\right] \\
& +M S_{L T \alpha} D_{L T}\left(z, k_{F \perp}\right)+k_{F \perp}^{\rho} S_{T T \rho \alpha} D_{T T}^{\perp \perp}\left(z, k_{F \perp}\right) \\
& +\frac{M^{2}}{p^{+}} n_{\alpha}\left[S_{L L} D_{3 L L}\left(z, k_{F \perp}\right)+\frac{k_{F \perp} \cdot S_{L T}}{M} D_{3 L T}^{\perp}\left(z, k_{F \perp}\right)+\frac{k_{F \perp} \cdot S_{T T} \cdot k_{F \perp}}{M^{2}} D_{3 T T}^{\perp}\left(z, k_{F \perp}\right)\right], \\
& z \tilde{\Xi}_{\alpha}^{T(0)}\left(z, k_{F \perp} ; p, S\right)=p^{+} \bar{n}_{\alpha}\left[\frac{\varepsilon_{\perp}^{k_{F \perp} S_{L T}}}{M} G_{1 L T}^{\perp}\left(z, k_{F \perp}\right)+\frac{\varepsilon_{\perp k_{F \perp} \rho} k_{F \perp \sigma} S_{T T}^{\rho \sigma}}{M^{2}} G_{1 T T}^{\perp}\left(z, k_{F \perp}\right)\right] \\
& +\varepsilon_{\perp \rho \alpha} k_{F \perp}^{\rho}\left[S_{L L} G_{L L}^{\perp}\left(z, k_{F \perp}\right)+\frac{k_{F \perp} \cdot S_{L T}}{M} G_{L T}^{\perp}\left(z, k_{F \perp}\right)+\frac{k_{F \perp} \cdot S_{T T} \cdot k_{F \perp}}{M^{2}} G_{T T}^{\perp}\left(z, k_{F \perp}\right)\right] \\
& +M \varepsilon_{\perp \rho \alpha} S_{L T}^{\rho} G_{L T}\left(z, k_{F \perp}\right)+\varepsilon_{\perp \alpha \rho} k_{F \perp \sigma} S_{T T}^{\rho \sigma} G_{T T}^{\prime \perp}\left(z, k_{F \perp}\right) \\
& +\frac{M^{2}}{p^{+}} n_{\alpha}\left[\frac{\varepsilon_{\perp}^{k_{F \perp} S_{L T}}}{M} G_{3 L T}^{\perp}\left(z, k_{F \perp}\right)+\frac{\varepsilon_{\perp k_{F \perp} \rho} k_{F \perp \sigma} S_{T T}^{\rho \sigma}}{M^{2}} G_{3 T T}^{\perp}\left(z, k_{F \perp}\right)\right] \\
& z \Xi_{T \rho \alpha}^{T(0)}\left(z, k_{F \perp} ; p, S\right)=\frac{p^{+} \bar{n}_{[\rho} \varepsilon_{\perp \alpha] \sigma} k_{F \perp}^{\sigma}}{M}\left[S_{L L} H_{1 L L}^{\perp}\left(z, k_{F \perp}\right)+\frac{k_{F \perp} \cdot S_{L T}}{M} H_{1 L T}^{\perp}\left(z, k_{F \perp}\right)\right. \\
& \left.+\frac{k_{F \perp} \cdot S_{T T} \cdot k_{F \perp}}{M^{2}} H_{1 T T}^{\perp}\left(z, k_{F \perp}\right)\right]+p^{+} \bar{n}_{\left[\rho \varepsilon_{\perp \alpha] \sigma}\right.} S_{L T}^{\sigma} H_{1 L T}\left(z, k_{F \perp}\right)+\frac{p^{+} \bar{n}_{[\rho} \varepsilon_{\perp \alpha] \sigma} k_{F \perp \delta} S_{T T}^{\sigma \delta}}{M} H_{1 T T}^{\prime \perp}\left(z, k_{F \perp}\right) \\
& +M \varepsilon_{\perp \rho \alpha}\left[S_{L L} H_{L L}\left(z, k_{F \perp}\right)+\frac{k_{F \perp} \cdot S_{L T}}{M} H_{L T}^{\perp}\left(z, k_{F \perp}\right)+\frac{k_{F \perp} \cdot S_{T T} \cdot k_{F \perp}}{M^{2}} H_{T T}^{\perp}\left(z, k_{F \perp}\right)\right] \\
& +\bar{n}_{[\rho} n_{\alpha]}\left[\varepsilon_{\perp}^{k_{F \perp} S_{L T}} H_{L T}^{\prime \perp}\left(z, k_{F \perp}\right)+\frac{\varepsilon_{\perp k_{F \perp} \sigma} k_{F \perp \delta} S_{T T}^{\sigma \delta}}{M} H_{T T}^{\prime \perp}\left(z, k_{F \perp}\right)\right]
\end{aligned}
$$




$$
\begin{aligned}
& +\frac{M^{2}}{p^{+}}\left\{\frac{n_{[\rho} \varepsilon_{\perp \alpha] \sigma} k_{F \perp}^{\sigma}}{M}\left[S_{L L} H_{3 L L}^{\perp}\left(z, k_{F \perp}\right)+\frac{k_{F \perp} \cdot S_{L T}}{M} H_{3 L T}^{\perp}\left(z, k_{F \perp}\right)+\frac{k_{F \perp} \cdot S_{T T} \cdot k_{F \perp}}{M^{2}} H_{3 T T}^{\perp}\left(z, k_{F \perp}\right)\right]\right. \\
& \left.+n_{[\rho} \varepsilon_{\perp \alpha] \sigma} S_{L T}^{\sigma} H_{3 L T}\left(z, k_{F \perp}\right)+\frac{n_{[\rho} \varepsilon_{\perp \alpha] \sigma} k_{F \perp \delta} S_{T T}^{\sigma \delta}}{M} H_{3 T T}^{\prime \perp}\left(z, k_{F \perp}\right)\right\} .
\end{aligned}
$$

We see that, for the vector-polarization-dependent part, similar to case of the nucleon TMD PDFs, there are a total of 32 components; 8 contribute at leading twist, 16 contribute at twist-3, and the other 8 contribute at twist- 4 . For the tensor-polarization-dependent part, there are a total of 40 components, where 10 contribute at leading twist, 20 contribute at twist-3, and the other 10 contribute at twist- 4 . In Table 4, we list the twist- 2 components for the tensor-polarization-dependent part.

If we integrate over $\mathrm{d}^{2} k_{F \perp}$, we have the following, corresponding to Eqs. (3.12)-(3.16), for the spin-independent and vector-polarization-dependent parts:

$$
\begin{aligned}
& z \Xi_{S}^{U+V(0)}(z ; p, S)=M E(z), \\
& z \Xi_{P S}^{U+V(0)}(z ; p, S)=\lambda M E_{L}(z), \\
& z \Xi_{\alpha}^{U+V(0)}(z ; p, S)=p^{+} \bar{n}_{\alpha} D_{1}(z)+M \varepsilon_{\perp \alpha \rho} S_{T}^{\rho} D_{T}(z)+\frac{M^{2}}{p^{+}} n_{\alpha} D_{3}(z), \\
& z \tilde{\Xi}_{\alpha}^{U+V(0)}(z ; p, S)=\lambda p^{+} \bar{n}_{\alpha} G_{1 L}(z)+M S_{T \alpha} G_{T}(z)+\lambda \frac{M^{2}}{p^{+}} n_{\alpha} G_{3 L}(z), \\
& z \Xi_{T \rho \alpha}^{U+V(0)}(z ; p, S)=p^{+} \bar{n}_{[\rho} S_{T \alpha]} H_{1 T}(z)+M \varepsilon_{\perp \rho \alpha} H(z)+\lambda M \bar{n}_{[\rho} n_{\alpha]} H_{L}(z)+\frac{M^{2}}{p^{+}} n_{[\rho} S_{T \alpha]} H_{3 T}(z) .
\end{aligned}
$$

For the tensor-polarization-dependent part, we have

$$
\begin{aligned}
& z \Xi_{S}^{T(0)}(z ; p, S)=M S_{L L} E_{L L}(z), \\
& z \Xi_{P S}^{T(0)}(z ; p, S)=0, \\
& z \Xi_{\alpha}^{T(0)}(z ; p, S)=p^{+} \bar{n}_{\alpha} S_{L L} D_{1 L L}(z)+M S_{L T \alpha} D_{L T}\left(z, k_{F \perp}\right)+\frac{M^{2}}{p^{+}} n_{\alpha} S_{L L} D_{3 L L}(z), \\
& z \tilde{\Xi}_{\alpha}^{T(0)}(z ; p, S)=M \varepsilon_{\perp \rho \alpha} S_{L T}^{\rho} G_{L T}(z), \\
& z \Xi_{T \rho \alpha}^{T(0)}(z ; p, S)=p^{+} \bar{n}_{[\rho} \varepsilon_{\perp \alpha] \sigma} S_{L T}^{\sigma} H_{1 L T}(z)+M \varepsilon_{\perp \rho \alpha} S_{L L} H_{L L}(z)+\frac{M^{2}}{p^{+}} n_{[\rho} \varepsilon_{\perp \alpha] \sigma} S_{L T}^{\sigma} H_{3 L T}(z) .
\end{aligned}
$$

\begin{tabular}{|c|c|c|c|c|}
\hline Parameters & $b_{11}$ & $b_{22}$ & $b_{12}$ & \\
\hline $\begin{array}{c}\text { Quark } \\
\text { polarization }\end{array}$ & $\begin{array}{c}\text { Hadron } \\
\text { polarization }\end{array}$ & TMD FFs & Integrated over $\vec{k}_{F \perp}$ & Name \\
\hline \multirow{2}{*}{$U$} & $L L$ & $D_{1 L L}\left(z, k_{F \perp}\right)$ & $D_{1 L L}(z)$ & Spin alignment \\
\hline & $T T$ & $D_{1 T T}^{\perp}\left(z, k_{F \perp}\right)$ & $\times$ & \\
\hline$L$ & $L T$ & $G_{1 L T}^{\perp}\left(z, k_{F \perp}\right)$ & $x$ & \\
\hline \multirow[t]{2}{*}{$T$} & $L T$ & $H_{1 L T}\left(z, k_{F \perp}\right), H_{1 L T}^{\perp}\left(z, k_{F \perp}\right)$ & $H_{1 L T}(z)$ & \\
\hline & $T T$ & $H_{1 T T}^{\perp}\left(z, k_{F \perp}\right), H_{1 T T}^{\prime \perp}\left(z, k_{F \perp}\right)$ & $x$ & \\
\hline
\end{tabular}

Table 4 The 10 tensor polarization dependent TMD FFs for spin- 1 hadrons defined via the quark-quark correlator. A $\times$ means that the corresponding term disappears upon integrating the quark-quark correlator over $d^{2} k_{F \perp}$.

We see that, for the spin-independent and vectorpolarization-dependent parts, 12 components survive; 3 of them contribute at twist-2, 6 contribute at twist-3, and the other 3 contribute at twist- 4 . This is exactly the same as the result for the PDFs for nucleons, and we have exact one-to-one correspondence between the results given by Eqs. (3.22)-(3.26) and those given by Eqs. (2.64)-
(2.68). For the tensor-polarization-dependent part, only 8 components survive; 2 of them contribute at twist- 2,4 contribute at twist- 3 , and the other 2 contribute at twist4. This corresponds to the situation for the PDFs for vector mesons. We should have a one-to-one correspondence between the tensor-polarization-dependent FFs for production of spin-1 hadrons and those PDFs for spin-1 
hadrons. We also list the twist-2 components in Table 4.

\section{Accessing the TMDs in high-energy reactions}

The TMDs can be studied in semi-inclusive high-energy reactions such as SIDIS $\left(e^{-}+N \rightarrow e^{-}+h+X\right)$, semiinclusive Drell-Yan processes $\left(h+h \rightarrow l^{+}+l^{-}+X\right)$, and semi-inclusive hadron production in $e^{+} e^{-}$-annihilation $\left(e^{+}+e^{-} \rightarrow h_{1}+h_{2}+X\right)$. For SIDIS, we study TMD PDFs and TMD FFs, whereas for Drell-Yan processes and $e^{+} e^{-}$annihilation, we study TMD PDFs and TMD FFs separately. We now follow the same steps as those for inclusive DIS and summarize briefly what we have already done in constructing the corresponding theoretical framework.

(I) The general forms of hadronic tensors: For all three classes of processes, the general forms of hadronic tensors have been studied and obtained. For SIDIS, this has been discussed in Refs. [27-30], and it has been shown that one needs 18 independent structure functions for spinless $h$. A comprehensive study of Drell-Yan processes was made in Ref. [31], and the number of independent structure functions is 48 for hadrons with spin $1 / 2$. A study of $e^{+} e^{-}$annihilation was presented in Ref. [32], and one needs 72 for spin- $1 / 2 h_{1}$ and $h_{2}$. The results are systematically presented in those papers, and we will not repeat them here. However, we present, as an example, the general form of the differential cross section for $e^{-} N \rightarrow e^{-} h X$. It is given by

$$
\begin{aligned}
& \frac{\mathrm{d} \sigma}{\mathrm{d} x \mathrm{~d} y \mathrm{~d} z \mathrm{~d} \psi \mathrm{d}^{2} p_{h \perp}}=\frac{\alpha_{e m}^{2}}{x y Q^{2}}\left(1+\frac{\gamma^{2}}{2 x}\right)\left(\mathcal{F}_{U U}+\lambda_{l} \mathcal{F}_{L U}+\lambda \mathcal{F}_{U L}+\lambda_{l} \lambda \mathcal{F}_{L L}+S_{\perp} \mathcal{F}_{U T}+\lambda_{l} S_{\perp} \mathcal{F}_{L T}\right), \\
& \mathcal{F}_{U U}=\frac{y^{2}}{1-\varepsilon}\left(F_{U U, T}+\varepsilon F_{U U, L}+\sqrt{2 \varepsilon(1+\varepsilon)} F_{U U}^{\cos \phi_{h}} \cos \phi_{h}+\varepsilon F_{U U}^{\cos 2 \phi_{h}} \cos 2 \phi_{h}\right), \\
& \mathcal{F}_{U L}=\frac{y^{2}}{1-\varepsilon}\left(\sqrt{2 \varepsilon(1+\varepsilon)} F_{U L}^{\sin \phi_{h}} \sin \phi_{h}+\varepsilon F_{U L}^{\sin 2 \phi_{h}} \sin 2 \phi_{h}\right), \\
& \mathcal{F}_{L U}=\frac{y^{2}}{1-\varepsilon} \sqrt{2 \varepsilon(1-\varepsilon)} F_{L U}^{\sin \phi_{h}} \sin \phi_{h}, \\
& \mathcal{F}_{L L}=\frac{y^{2}}{1-\varepsilon}\left(\sqrt{1-\varepsilon^{2}} F_{L L}+\sqrt{2 \varepsilon(1-\varepsilon)} F_{L L}^{\cos \phi_{h}} \cos \phi_{h}\right), \\
& \mathcal{F}_{U T}=\frac{y^{2}}{1-\varepsilon}\left[\sqrt{2 \varepsilon(1+\varepsilon)} F_{U T}^{\sin \phi_{S}} \sin \phi_{S}+\left(F_{U T, T}^{\sin \left(\phi_{h}-\phi_{S}\right)}+\varepsilon F_{U T, L}^{\sin \left(\phi_{h}-\phi_{S}\right)}\right) \sin \left(\phi_{h}-\phi_{S}\right)\right. \\
&\left.\quad+\varepsilon F_{U T}^{\sin \left(\phi_{h}+\phi_{S}\right)} \sin \left(\phi_{h}+\phi_{S}\right)+\sqrt{2 \varepsilon(1+\varepsilon)} F_{U T}^{\sin \left(2 \phi_{h}-\phi_{S}\right)} \sin \left(2 \phi_{h}-\phi_{S}\right)+\varepsilon F_{U T}^{\sin \left(3 \phi_{h}-\phi_{S}\right)} \sin \left(3 \phi_{h}-\phi_{S}\right)\right], \\
& \mathcal{F}_{L T}= \frac{y^{2}}{1-\varepsilon}\left[\sqrt{2 \varepsilon(1-\varepsilon)} F_{L T}^{\cos \phi_{S}} \cos \phi_{S}+\sqrt{1-\varepsilon^{2}} F_{L T}^{\cos \left(\phi_{h}-\phi_{S}\right)} \cos \left(\phi_{h}-\phi_{S}\right)\right. \\
&\left.+\sqrt{2 \varepsilon(1-\varepsilon)} F_{L T}^{\cos \left(2 \phi_{h}-\phi_{S}\right)} \cos \left(2 \phi_{h}-\phi_{S}\right)\right]
\end{aligned}
$$

where $\varepsilon=\left(1-y-\frac{1}{4} \gamma^{2} y^{2}\right) /\left(1-y+\frac{1}{2} y^{2}+\frac{1}{4} \gamma^{2} y^{2}\right)$, $\gamma=2 M x / Q$; the azimuthal angle $\psi$ is that of the outgoing lepton $\vec{l}^{\prime}$ around the incident lepton beam with respect to an arbitrary fixed direction, which, for a transversely polarized target, is taken as the direction of $\vec{S}_{T}$. In the deep inelastic limit, neglecting power-suppressed terms, $\mathrm{d} \psi=\mathrm{d} \phi_{S}$.

From Eqs. (4.1)-(4.7), we see explicitly that the 18 structure functions $F$ are determined by the different azimuthal asymmetries in different polarization cases. These different azimuthal asymmetries are just defined by the average value of the corresponding trigonometric functions, for example:

$$
\begin{aligned}
A_{U T}^{\sin \left(\phi_{h}-\phi_{S}\right)} & =\left\langle\sin \left(\phi_{h}-\phi_{S}\right)\right\rangle_{U T} \\
& =\frac{F_{U T, T}^{\sin \left(\phi_{h}-\phi_{S}\right)}+\varepsilon F_{U T, L}^{\sin \left(\phi_{h}-\phi_{S}\right)}}{2\left(F_{U U, T}+\varepsilon F_{U U, L}\right)},
\end{aligned}
$$

$$
\begin{aligned}
A_{U T}^{\sin \left(\phi_{h}+\phi_{S}\right)} & =\left\langle\sin \left(\phi_{h}+\phi_{S}\right)\right\rangle_{U T} \\
& =\frac{\varepsilon F_{U T}^{\sin \left(\phi_{h}+\phi_{S}\right)}}{2\left(F_{U U, T}+\varepsilon F_{U U, L}\right)}
\end{aligned}
$$

We also emphasize that they are the general forms independent of the parton model and are valid at leading and higher twist and also leading and higher order in pQCD.

(II) LO in pQCD and leading twist parton model results: These are the simplest parton model results and can be obtained easily. E.g., for SIDIS,

$$
\begin{gathered}
\frac{\mathrm{d} \sigma^{(0)}}{\mathrm{d} x \mathrm{~d} y \mathrm{~d} z \mathrm{~d} \phi_{S} \mathrm{~d}^{2} p_{h \perp}}=\frac{\alpha_{e m}^{2}}{x y Q^{2}}\left(\mathcal{F}_{U U}^{(0)}+\lambda_{l} \mathcal{F}_{L U}^{(0)}+\lambda \mathcal{F}_{U L}^{(0)}\right. \\
\left.+\lambda_{l} \lambda \mathcal{F}_{L L}^{(0)}+S_{\perp} \mathcal{F}_{U T}^{(0)}+\lambda_{l} S_{\perp} \mathcal{F}_{L T}^{(0)}\right), \\
\mathcal{F}_{U U}^{(0)}=
\end{gathered}
$$




$$
\begin{aligned}
& \text { (4.11) } \mathcal{F}_{L U}^{(0)}=0 \text {, } \\
& \mathcal{F}_{U L}^{(0)}=2(1-y) \mathcal{C}\left[w_{1} h_{1 L}^{\perp} H_{1}^{\perp}\right] \sin \left(2 \phi_{h}\right), \\
& \text { (4.12) } \quad \mathcal{F}_{L L}^{(0)}=C(y) \mathcal{C}\left[g_{1 L} D_{1}\right] \\
& \mathcal{F}_{U T}^{(0)}=A(y) \mathcal{C}\left[w_{2} f_{1 T}^{\perp} D_{1}\right] \sin \left(\phi_{h}-\phi_{S}\right)+2(1-y) \mathcal{C}\left[w_{3} h_{1 T} H_{1}^{\perp}\right] \sin \left(\phi_{h}+\phi_{S}\right)+2(1-y) \mathcal{C}\left[w_{4} h_{1 T}^{\perp} H_{1}^{\perp}\right] \sin \left(3 \phi_{h}-\phi_{S}\right), \\
& \mathcal{F}_{L T}^{(0)}=C(y) \mathcal{C}\left[-w_{2} g_{1 T} D_{1}\right] \cos \left(\phi_{h}-\phi_{S}\right),
\end{aligned}
$$

where $A(y)=1+(1-y)^{2}$, and $C(y)=y(2-y) \cdot \mathcal{C}\left[w_{i} f D\right]$ denotes the convolution of $f$ and $D$ weighted by $w_{i}$, i.e.,

$$
\mathcal{C}\left[w_{i} f D\right] \equiv x \sum_{q} e_{q}^{2} \int d^{2} k_{\perp} d^{2} k_{F \perp} \delta^{(2)}\left(k_{\perp}-k_{F \perp}-p_{h T} / z\right) w_{i}\left(k_{\perp}, k_{F \perp}, p_{h T}\right) f^{q}\left(x, k_{\perp}\right) D^{q \rightarrow h X}\left(z, k_{F \perp}\right) .
$$

The weights $w_{i}$ are given by

$$
\begin{aligned}
& w_{1}\left(\vec{k}_{\perp}, k_{F \perp}\right)=\frac{-2\left(\hat{p}_{h T} \cdot \vec{k}_{F \perp}\right)\left(\hat{p}_{h T} \cdot \vec{k}_{\perp}\right)+\left(\vec{k}_{\perp} \cdot \vec{k}_{F \perp}\right)}{M M_{h}}, \\
& w_{2}\left(\vec{k}_{\perp}, k_{F \perp}\right)=-\frac{\hat{p}_{h T} \cdot \vec{k}_{\perp}}{M}, \\
& w_{3}\left(\vec{k}_{\perp}, k_{F \perp}\right)=-\frac{\hat{p}_{h T} \cdot \vec{k}_{F \perp}}{M_{h}}, \\
& w_{4}\left(\vec{k}_{\perp}, k_{F \perp}\right)=\frac{\left(\hat{p}_{h T} \cdot \vec{k}_{\perp}\right)\left(\vec{k}_{\perp} \cdot \vec{k}_{F \perp}\right)+\vec{k}_{\perp}^{2}\left(\hat{p}_{h T} \cdot \vec{k}_{F \perp}\right)}{M^{2} M_{h}} \\
& -\frac{2\left(\hat{p}_{h T} \cdot \vec{k}_{\perp}\right)^{2}\left(\hat{p}_{h T} \cdot \vec{k}_{F \perp}\right)}{M^{2} M_{h}},
\end{aligned}
$$

where $\hat{p}_{h T}=\vec{p}_{h T} /\left|\vec{p}_{h T}\right|$ is the corresponding unit vector. The results can be obtained from those given in, e.g., [30] by neglecting all the power-suppressed contributions.

From Eqs. (4.10)-(4.16), we see in particular that, at leading twist, there exist six nonzero azimuthal asymmetries in different polarization cases, i.e.,

$$
\begin{aligned}
& \left\langle\cos 2 \phi_{h}\right\rangle_{U U}^{(0)}=\frac{(1-y)}{A(y)} \frac{\mathcal{C}\left[w_{1} h_{1}^{\perp} H_{1}^{\perp}\right]}{\mathcal{C}\left[f_{1} D_{1}\right]}, \\
& \left\langle\sin 2 \phi_{h}\right\rangle_{U L}^{(0)}=\frac{(1-y)}{A(y)} \frac{\mathcal{C}\left[w_{1} h_{1 L}^{\perp} H_{1}^{\perp}\right]}{\mathcal{C}\left[f_{1} D_{1}\right]} \\
& \left\langle\sin \left(\phi_{h}-\phi_{S}\right)\right\rangle_{U T}^{(0)}=\frac{\mathcal{C}\left[w_{2} f_{1 T}^{\perp} D_{1}\right]}{2 \mathcal{C}\left[f_{1} D_{1}\right]} \\
& \left\langle\sin \left(\phi_{h}+\phi_{S}\right)\right\rangle_{U T}^{(0)}=\frac{(1-y)}{A(y)} \frac{\mathcal{C}\left[w_{3} h_{1 T} H_{1}^{\perp}\right]}{\mathcal{C}\left[f_{1} D_{1}\right]} \\
& \left\langle\sin \left(3 \phi_{h}-\phi_{S}\right)\right\rangle_{U T}^{(0)}=\frac{(1-y)}{A(y)} \frac{\mathcal{C}\left[w_{4} h_{1 T}^{\perp} H_{1}^{\perp}\right]}{\mathcal{C}\left[f_{1} D_{1}\right]}
\end{aligned}
$$

$$
\left\langle\cos \left(\phi_{h}-\phi_{S}\right)\right\rangle_{L T}^{(0)}=\frac{C(y)}{2 A(y)} \frac{\mathcal{C}\left[-w_{2} g_{1 T} D_{1}\right]}{\mathcal{C}\left[f_{1} D_{1}\right]} .
$$

They are determined by the Boer-Mulders function $h_{1}^{\perp}$ convoluted with the Collins function $H_{1}^{\perp}$, the wormgear (longi-transversity) $h_{1 L}^{\perp}$ convoluted with the Collins function $H_{1}^{\perp}$, the Sivers function $f_{1 T}^{\perp}$ convoluted with $D_{1}$, the transversity $h_{1 T}$ convoluted with the Collins function $H_{1}^{\perp}$, and the worm-gear (trans-helicity distribution) $g_{1 T}^{\perp}$ convoluted with the Collins function $H_{1}^{\perp}$. The azimuthal asymmetries $A_{U T}^{\sin \left(\phi_{h} \mp \phi_{S}\right)}$ are due to the Sivers and Collins effects and are often referred to as Sivers asymmetry and Collins asymmetry, respectively.

We emphasize that the results given by Eqs. (4.10)(4.21) give a complete parton model result at LO in pQCD and leading twist. They can be used to extract the TMDs at this order. Any attempt to go beyond LO in $\mathrm{pQCD}$ or to consider higher twists needs to go beyond this expression.

(III) LO in pQCD, leading and higher twist results: For semi-inclusive processes in which only one hadron is involved, either in the initial or the final state, it has been shown [33-37] that collinear expansion can be applied. Such processes include SIDIS $\left[e^{-}+N \rightarrow e^{-}+q(\right.$ jet $\left.)+X\right]$, and $e^{+} e^{-}$-annihilation $\left[e^{+}+e^{-} \rightarrow h+\bar{q}(j e t)+X\right]$. By applying collinear expansion, we have constructed the theoretical framework for these processes by which leading as well as higher twist contributions can be calculated systematically to LO in pQCD. The complete results up to twist-3 are presented in Refs. [35-37]. For polarized $e^{-}+N \rightarrow e^{-}+q(j e t)+X$, the simplified expressions for the hadronic tensor are very similar to those for the inclusive DIS given by Eqs. (2.55)-(2.58),

$$
\begin{aligned}
& \tilde{W}_{\mu \nu}^{(0, s i)}\left(q, p, S, k_{\perp}\right)=\frac{1}{2} \operatorname{Tr}\left[\hat{h}_{\mu \nu}^{(0)} \hat{\Phi}^{(0)}\left(x_{B}, k_{\perp}\right)\right], \\
& \tilde{W}_{\mu \nu}^{(1, L, s i)}\left(q, p, S, k_{\perp}\right)=\frac{1}{4 q \cdot p} \operatorname{Tr}\left[\hat{h}_{\mu \nu}^{(1) \rho} \omega_{\rho}^{\rho^{\prime}} \hat{\varphi}_{\rho^{\prime}}^{(1, L)}\left(x_{B}, k_{\perp}\right)\right],
\end{aligned}
$$




$$
\begin{aligned}
& \tilde{W}_{\mu \nu}^{(2, L, s i)}\left(q, p, S, k_{\perp}\right)=\frac{1}{(2 q \cdot p)^{2}}\left\{\operatorname{Tr}\left[\hat{h}_{\mu \nu}^{(1) \rho} \omega_{\rho}^{\rho^{\prime}} \hat{\phi}_{\rho^{\prime}}^{(2 L)}\left(x_{B}, k_{\perp}\right)\right]+\operatorname{Tr}\left[\hat{N}_{\mu \nu}^{(2) \rho \sigma} \omega_{\rho}^{\rho^{\prime}} \omega_{\sigma}^{\sigma^{\prime}} \hat{\varphi}_{\rho^{\prime} \sigma^{\prime}}^{(2 L)}\left(x_{B}, k_{\perp}\right)\right]\right\}, \\
& \tilde{W}_{\mu \nu}^{(2, M, s i)}\left(q, p, S, k_{\perp}\right)=\frac{1}{(2 q \cdot p)^{2}} \operatorname{Tr}\left[\hat{h}_{\mu \nu}^{(2) \rho \sigma} \omega_{\rho}^{\rho^{\prime}} \omega_{\sigma}^{\sigma^{\prime}} \hat{\varphi}_{\rho^{\prime} \sigma^{\prime}}^{(2 M)}\left(x_{B}, k_{\perp}\right)\right] .
\end{aligned}
$$

The complete results up to twist-3 are given by

$$
\begin{aligned}
& \frac{\mathrm{d} \sigma}{\mathrm{d} x \mathrm{~d} y \mathrm{~d}^{2} k_{\perp}}=\frac{2 \pi \alpha_{e m}^{2} e_{q}^{2}}{Q^{2} y}\left(\mathcal{W}_{U U}+\lambda_{l} \mathcal{W}_{L U}+S_{\perp} \mathcal{W}_{U T}+\lambda \mathcal{W}_{U L}+\lambda_{l} \lambda \mathcal{W}_{L L}+\lambda_{l} S_{\perp} \mathcal{W}_{L T}\right), \\
& \mathcal{W}_{U U}\left(x, k_{\perp}, \phi\right)=A(y) f_{q}\left(x, k_{\perp}\right)-\frac{2 x\left|\vec{k}_{\perp}\right|}{Q} B(y) f_{q}^{\perp}\left(x, k_{\perp}\right) \cos \phi, \\
& \mathcal{W}_{L U}\left(x, k_{\perp}, \phi\right)=-\frac{2 x\left|\vec{k}_{\perp}\right|}{Q} D(y) g^{\perp}\left(x, k_{\perp}\right) \sin \phi, \\
& \mathcal{W}_{U T}\left(x, k_{\perp}, \phi, \phi_{S}\right)=\frac{\left|\vec{k}_{\perp}\right|}{M} A(y) f_{1 T}^{\perp}\left(x, k_{\perp}\right) \sin \left(\phi-\phi_{S}\right) \\
& \quad+\frac{2 x M}{Q} B(y)\left\{\frac{k_{\perp}^{2}}{2 M^{2}} f_{T}^{\perp}\left(x, k_{\perp}\right) \sin \left(2 \phi-\phi_{S}\right)-f_{T}\left(x, k_{\perp}\right) \sin \phi_{S}\right\}, \\
& \mathcal{W}_{U L}\left(x, k_{\perp}, \phi\right)=-\frac{2 x\left|\vec{k}_{\perp}\right|}{Q} B(y) f_{L}^{\perp}\left(x, k_{\perp}\right) \sin \phi \\
& \mathcal{W}_{L L}\left(x, k_{\perp}, \phi\right)=C(y) g_{1 L}\left(x, k_{\perp}\right)-\frac{2 x\left|\vec{k}_{\perp}\right|}{Q} D(y) g_{L}^{\perp}\left(x, k_{\perp}\right) \cos \phi, \\
& \mathcal{W}_{L T}\left(x, k_{\perp}, \phi, \phi\right)=\frac{\left|\vec{k}_{\perp}\right|}{M} C(y) g_{1 T}^{\perp}\left(x, k_{\perp}\right) \cos \left(\phi-\phi_{S}\right) \\
& \quad-\frac{2 x M}{Q} D(y)\left[g_{T}\left(x, k_{\perp}\right) \cos \phi_{S}-\frac{k_{\perp}^{2}}{2 M^{2}} g_{T}^{\perp}\left(x, k_{\perp}\right) \cos \left(2 \phi-\phi_{S}\right)\right]
\end{aligned}
$$

where $B(y)=2(2-y) \sqrt{1-y}$, and $D(y)=2 y \sqrt{1-y}$. For unpolarized $e^{-}+N \rightarrow e^{-}+q(j e t)+X$, the results up to twist-4 have also been obtained [34]:

$$
\begin{aligned}
\frac{\mathrm{d} \sigma_{U U}}{\mathrm{~d} x \mathrm{~d} y \mathrm{~d}^{2} k_{\perp}}= & \frac{2 \pi \alpha_{e m}^{2} e_{q}^{2}}{Q^{2} y}\left\{A(y) f_{1}\left(x, k_{\perp}\right)-2 B(y) \frac{\left|\vec{k}_{\perp}\right|}{Q} x f^{\perp}\left(x, k_{\perp}\right) \cos \phi\right. \\
& -4(1-y) \frac{\left|\vec{k}_{\perp}\right|^{2}}{Q^{2}} x\left[\varphi_{3}^{(1) \perp}\left(x, k_{\perp}\right)-\tilde{\varphi}_{3}^{(1) \perp}\left(x, k_{\perp}\right)\right] \cos 2 \phi \\
& \left.+8(1-y) \frac{2 x^{2} M^{2}}{Q^{2}} f_{3}\left(x, k_{\perp}\right)-2 A(y) \frac{\left|\vec{k}_{\perp}\right|^{2}}{Q^{2}} x\left[\varphi_{3}^{(2, L) \perp}\left(x, k_{\perp}\right)-\tilde{\varphi}_{3}^{(2, L) \perp}\left(x, k_{\perp}\right)\right]\right\} .
\end{aligned}
$$

These results are expressed in terms of the gauge-invariant TMD PDFs or FFs and can be used as the basis for measuring these TMDs via the corresponding process at the LO in pQCD.

We would like in particular to draw attention to the results for $e^{+}+e^{-} \rightarrow h+\bar{q}(j e t)+X$ for $h$ with different spins [37]. Here, for the hadronic tensor, we again obtain very similar formulae for this process; e.g., corresponding to Eqs. (4.28)-(4.30), we have

$$
\begin{aligned}
& \tilde{W}_{\mu \nu}^{(0, s i)}\left(q, p, S, k_{\perp}^{\prime} \mid e^{+} e^{-}\right)=\frac{1}{2} \operatorname{Tr}\left[\hat{h}_{\mu \nu}^{(0)} \hat{\Xi}^{(0)}\left(z_{B}, k_{\perp}^{\prime}\right)\right], \\
& \tilde{W}_{\mu \nu)}^{(1, L, s i)}\left(q, p, S, k_{\perp}^{\prime} \mid e^{+} e^{-}\right)=-\frac{1}{4 p \cdot q} \operatorname{Tr}\left[\hat{h}_{\mu \nu}^{(1) \rho} \omega_{\rho}^{\rho^{\prime}} \hat{\Xi}_{\rho^{\prime}}^{(1)}\left(z_{B}, k_{\perp}^{\prime}\right)\right], \\
& \tilde{W}_{\mu \nu}^{(2, L, s i)}\left(q, p, S, k_{\perp}^{\prime} \mid e^{+} e^{-}\right)=\frac{1}{4(p \cdot q)^{2}} \operatorname{Tr}\left[\hat{h}_{\mu \nu}^{(1) \rho} \omega_{\rho}^{\rho^{\prime}} \hat{\Xi}_{\rho^{\prime}}^{(2 B)}\left(z_{B}, k_{\perp}^{\prime}\right)+\hat{N}_{\mu \nu}^{(2) \rho \sigma} \omega_{\rho}^{\rho^{\prime}} \omega_{\sigma}^{\sigma^{\prime}} \hat{\Xi}_{\rho^{\prime} \sigma^{\prime}}^{(2 C)}\left(z_{B}, k_{\perp}^{\prime}\right)\right], \\
& \tilde{W}_{\mu \nu}^{(2, M, s i)}\left(q, p, S, k_{\perp}^{\prime} \mid e^{+} e^{-}\right)=\frac{1}{4(p \cdot q)^{2}} \operatorname{Tr}\left[\hat{h}_{\mu \nu}^{(2) \rho \sigma} \omega_{\rho}^{\rho^{\prime}} \omega_{\sigma}^{\sigma^{\prime}} \hat{\Xi}_{\rho^{\prime} \sigma^{\prime}}^{(2 A)}\left(z_{B}, k_{\perp}^{\prime}\right)\right] .
\end{aligned}
$$


Complete twist-3 results for the differential cross sections, azimuthal asymmetries, and polarizations were obtained for hadrons with spin $0,1 / 2$, and 1 in Ref. [37]. For spin-1 hadrons in particular, we see that tensor polarization is involved, even at the leading twist level; we have, for $e^{+} e^{-}$annihilation at the $Z^{0}$ pole,

$$
\begin{aligned}
S_{L L}^{(0)}\left(y, z, p_{T}\right) & =\frac{\sum_{q} T_{0}^{q}(y) D_{1 L L}\left(z, p_{T}\right)}{2 \sum_{q} T_{0}^{q}(y) D_{1}\left(z, p_{T}\right)}, \\
S_{L T}^{n(0)}\left(y, z, p_{T}\right) & =-\frac{2\left|\vec{p}_{T}\right|}{3 z M} \frac{\sum_{q} P_{q}(y) T_{0}^{q}(y) G_{1 L T}^{\perp}\left(z, p_{T}\right)}{\sum_{q} T_{0}^{q}(y) D_{1}\left(z, p_{T}\right)},
\end{aligned}
$$

$$
\begin{aligned}
& S_{L T}^{t(0)}\left(y, z, p_{T}\right)=-\frac{2\left|\vec{p}_{T}\right|}{3 z M} \frac{\sum_{q} T_{0}^{q}(y) D_{1 L T}^{\perp}\left(z, p_{T}\right)}{\sum_{q} T_{0}^{q}(y) D_{1}\left(z, p_{T}\right)}, \\
& S_{T T}^{n n(0)}\left(y, z, p_{T}\right)=-\frac{2\left|\vec{p}_{T}\right|^{2}}{3 M^{2}} \frac{\sum_{q} T_{0}^{q}(y) D_{1 T T}^{\perp}\left(z, p_{T}\right)}{\sum_{q} T_{0}^{q}(y) D_{1}\left(y, p_{T}\right)}, \\
& S_{T T}^{n t(0)}\left(y, z, p_{T}\right)=\frac{2\left|\vec{p}_{T}\right|^{2}}{3 M^{2}} \frac{\sum_{q} P_{q}(y) T_{0}^{q}(y) G_{1 T T}^{\perp}\left(z, p_{T}\right)}{\sum_{q} T_{0}^{q}(y) D_{1}\left(y, p_{T}\right)},
\end{aligned}
$$

where $n$ and $t$ denote the two transverse directions of the produced vector meson, normal to and inside the production plane, respectively. The coefficient $T_{0}^{q}(y)=c_{1}^{q} c_{1}^{e}[(1-$ $\left.y)^{2}+y^{2}\right]-c_{3}^{q} c_{3}^{e}[1-2 y]$, where $c_{1}^{e}=\left(c_{V}^{e}\right)^{2}+\left(c_{A}^{e}\right)^{2}$, and $c_{3}^{e}=2 c_{V}^{e} c_{A}^{e} ; y$ in this reaction is defined as $y \equiv l_{1}^{+} / k^{+}$. $P_{q}(y)=T_{1}^{q}(y) / T_{0}^{q}(y)$ is the polarization of the quark produced by $Z^{0}$ decay, and $T_{1}^{q}(y)=-c_{3}^{q} c_{1}^{e}\left[(1-y)^{2}+\right.$ $\left.y^{2}\right]+c_{1}^{q} c_{3}^{e}[1-2 y]$. This situation has been much less explored to date and is a worthwhile topic for many further studies.

The three types of semi-inclusive processes mentioned above always involve two hadrons. How to apply collinear expansion to such processes has not been demonstrated. It is unclear how one can systematically calculate leading and higher twist contributions. Nevertheless, practical twist-3 calculations have been performed for these processes [38-41] using the following steps:

(i) draw Feynman diagrams with multiple gluon scattering to the order of one gluon exchange,

(ii) insert the gauge link in the correlator wherever needed to make it gauge invariant, and

(iii) carry out calculations to the order $1 / Q$.

Although this method has not been proved, it is interesting to see that the results obtained this way reduce exactly to those obtained in the corresponding simplified cases where collinear expansion is applied if we take the corresponding fragmentation functions as $\delta$ functions.

(IV) TMD factorization and evolution: To describe the semi-inclusive high-energy reactions mentioned above in terms of QCD and the parton model, TMDs are needed, and the factorization theorem has to involve the transverse momentum dependence. A TMD factorization theorem has been established at the leading twist for semi-inclusive processes [42-49]. TMD evolution theory is also developing very rapidly [50-63]. Boer [3] gave an overview at Spin2014, and an annual workshop series dedicated to this topic was established in 2012. We refer interested readers to these talks and overviews.

\section{Available data and parameterizations}

All three types of semi-inclusive reactions have been investigated experimentally. The results are summarized, e.g., in a number of plenary talks at Spin2014 by Stolarski and Rostomyan $[64,65]$. Here, we will just briefly summarize the main data available and then try to sort out the available TMD parameterizations.

For SIDIS, measurements have been made by the HERMES Collaboration [66-70] at DESY, the COMPASS Collaboration [71-78] at CERN, CLAS [79, 80], and the Hall A Collaboration [81-84] at Jefferson Laboratory (JLab). We list these SIDIS experiments in Table 5 and briefly summarize the results as follows.

At DESY, the single-spin asymmetries for SIDIS were first measured with a longitudinally polarized target by HERMES [66] for production of charged pions and then measured for the first time with a transversely polarized target in [67]. They found nonzero Sivers and Collins asymmetries $\left\langle\sin \left(\phi_{h}-\phi_{S}\right)\right\rangle_{U T}$ and $\left\langle\sin \left(\phi_{h}+\phi_{S}\right)\right\rangle_{U T}$. Measurements were then conducted for $\pi^{0}$ and kaons [68, 69] and also for azimuthal asymmetries $\left\langle\cos \phi_{h}\right\rangle_{U U}$ and $\left\langle\cos \left(2 \phi_{h}\right)\right\rangle_{U U}$ in the unpolarized case [70].

At CERN, COMPASS has measured the Sivers and Collins asymmetries in reactions with deuteron or proton targets for production of charged hadrons, pions, and kaons [71-78], and also $\left\langle\cos \phi_{h}\right\rangle_{U U}$ and $\left\langle\cos \left(2 \phi_{h}\right)\right\rangle_{U U}$ in the unpolarized case [78].

At JLab, CLAS has measured $[79,80]\left\langle\sin \left(2 \phi_{h}\right)\right\rangle_{U L}$ for pions with different charges and $\left\langle\sin \phi_{h}\right\rangle_{L U}$ for $\pi^{0}$. The Hall A Collaboration has measured [81-84] the Collins and Sivers asymmetries for $\pi^{ \pm}$and $K^{ \pm}$, and $\left\langle\cos \left(\phi_{h}-\phi_{s}\right)\right\rangle_{L T}$ for $\pi^{ \pm}$and $\left\langle\sin \left(3 \phi_{h}-\phi_{s}\right)\right\rangle_{U T}$. These measurements are all summarized in Table 5.

In addition to the data for SIDIS, we now have measurements of the azimuthal asymmetries in $e^{+} e^{-} \rightarrow$ $\pi+\pi+X$ made by Belle [85-87] and the BaBar collaboration [88], and also preliminary results from BES [89]. For Drell-Yan processes, data are available on the azimuthal asymmetries in, e.g., reactions using pion beams [90-93] 
and $p p$ or $p D$ collisions [94, 95].

Table 5 Available measurements on azimuthal asymmetries in SIDIS

\begin{tabular}{|c|c|c|c|}
\hline Collaboration & Reaction & Asymmetries & Ref.'s \\
\hline \multirow[t]{5}{*}{$\overline{\text { HERMES }}$} & $e^{+} N \rightarrow e^{+} \pi^{ \pm} X$ & $A_{U L}^{\sin \phi_{h}}, A_{U L}^{\sin 2 \phi_{h}}$ & {$[66]$} \\
\hline & $e^{+} N \rightarrow e^{+} \pi^{ \pm} X$ & $A_{\text {Siv }}, A_{\text {Coll }}$ & {$[67]$} \\
\hline & $e^{+} N \rightarrow e^{+} \pi^{ \pm, 0}\left(K^{ \pm}\right) X$ & $A_{S i v}$ & [68] \\
\hline & $e^{+} N \rightarrow e^{+} \pi^{ \pm, 0}\left(K^{ \pm}\right) X$ & $A_{\text {Coll }}$ & [69] \\
\hline & $e^{+} N \rightarrow e^{+} \pi^{ \pm}\left(K^{ \pm}\right) X$ & $A_{U U}^{\cos \phi_{h}}, A_{U U}^{\cos 2 \phi_{h}}$ & {$[70]$} \\
\hline \multirow[t]{7}{*}{$\overline{\text { COMPASS }}$} & $\mu^{-6} \mathrm{LiD} \rightarrow \mu^{-} h^{ \pm} X$ & $A_{\text {Siv }}, A_{\text {Coll }}$ & {$[71,72]$} \\
\hline & $\mu^{-6} \mathrm{LiD} \rightarrow \mu^{-} \pi^{ \pm}\left(K^{ \pm, 0}\right) \chi$ & $X A_{\text {Siv }}, A_{\text {Coll }}$ & {$[73]$} \\
\hline & $\mu^{-} \mathrm{NH}_{3} \rightarrow \mu^{-} h^{ \pm} X$ & $A_{\text {Siv }}, A_{\text {Coll }}$ & {$[74]$} \\
\hline & $\mu^{-} \mathrm{NH}_{3} \rightarrow \mu^{-} h^{ \pm} X$ & $A_{\text {Coll }}$ & {$[75]$} \\
\hline & $\mu^{-} \mathrm{NH}_{3} \rightarrow \mu^{-} h^{ \pm} X$ & $A_{S i v}$ & {$[76]$} \\
\hline & $\mu^{-} \mathrm{NH}_{3} \rightarrow \mu^{-} \pi^{ \pm}\left(K^{ \pm, 0}\right) X$ & $A_{\text {Siv }}, A_{\text {Coll }}$ & {$[77]$} \\
\hline & $\mu^{-6} \mathrm{LiD} \rightarrow \mu^{-} h^{ \pm} X$ & $A_{U U}^{\cos \phi_{h}}, A_{U U}^{\cos 2 \phi_{h}}$ & [78] \\
\hline \multirow[t]{2}{*}{$\overline{\mathrm{CLAS}}$} & $e^{-} p \rightarrow e^{-} \pi^{ \pm, 0} X$ & $A_{U L}^{\sin 2 \phi_{h}}$ & {$[79]$} \\
\hline & $e^{-} p \rightarrow e^{-} \pi^{0} X$ & $A_{L U}^{\sin \phi_{h}}$ & {$[80]$} \\
\hline \multirow[t]{4}{*}{ JLab Hall A } & $e^{-3} \mathrm{He} \rightarrow e^{-} \pi^{ \pm} X$ & $A_{\text {Siv }}, A_{\text {Coll }}$ & {$[81]$} \\
\hline & $e^{-3} \mathrm{He} \rightarrow e^{-} \pi^{ \pm} X$ & $A_{L T}^{\cos \left(\phi_{h}-\phi_{S}\right)}$ & [82] \\
\hline & $e^{-3} \mathrm{He} \rightarrow e^{-} \pi^{ \pm} X$ & $A_{U T}^{\sin \left(3 \phi_{h}-\phi_{S}\right)}$ & [83] \\
\hline & $e^{-3} \mathrm{He} \rightarrow e^{-} K^{ \pm} X$ & $A_{\text {Siv }}, A_{\text {Coll }}$ & {$[84]$} \\
\hline
\end{tabular}

Although the data are still far from sufficiently abundant to give precise control of the TMDs involved, different sets of TMD parameterizations have already been extracted from them. We briefly sort them out as follows.

The first part concerns what people called the firstphase parameterizations, i.e., TMD parameterizations without QCD evolution. Here, the following results in particular are available. We emphasize once more that all the results, including the figures, are taken from Refs. [96-112]. Interested readers are referred to these references for more details.

(1) Transverse momentum dependence: This is usually taken as [96-100] a Gaussian in a factorized form independent of the longitudinal variable $z$ or $x$, e.g.,

$$
\begin{aligned}
& f_{1}\left(x, k_{\perp}\right)=f_{1}(x) \mathrm{e}^{-\vec{k}_{\perp}^{2} /\left\langle\vec{k}_{\perp}^{2}\right\rangle} / \pi\left\langle\vec{k}_{\perp}^{2}\right\rangle, \\
& D_{1}\left(z, k_{F \perp}\right)=D_{1}(z) \mathrm{e}^{-\vec{k}_{F \perp}^{2} /\left\langle\vec{k}_{F \perp}^{2}\right\rangle} / \pi\left\langle\vec{k}_{F \perp}^{2}\right\rangle .
\end{aligned}
$$

The width has been fitted, and the form and flavor dependence and so on have been tested. The typical values of the fitted widths are, e.g., $\left\langle\vec{k}_{\perp}^{2}\right\rangle=0.25 \mathrm{GeV}^{2}$ and $\left\langle\vec{k}_{F \perp}^{2}\right\rangle=0.20 \mathrm{GeV}^{2}[96]$. Roughly speaking, this is a quite satisfactory fit. However, it has also been pointed out, e.g., in [99] for the TMD FF, that the Gaussian form seems to depend on the flavor and even on $z$, which means that it is only a zeroth-order approximation.

(2) Sivers function: All the data available from HERMES [67-69], COMPASS [71-74, 76, 77], and JLab Hall A $[81,82,84]$ on the Sivers asymmetries in SIDIS for pi- ons and kaons have been used for parameterization. The Sivers function is usually parameterized $[96,101-106]$ in the form of the number density $f_{q}\left(x, k_{\perp}\right)$ multiplied by an $x$-dependent factor $\mathcal{N}_{q}(x)$ and a $k_{\perp}$-dependent factor $h\left(k_{\perp}\right)$, i.e.,

$$
\Delta^{N} f_{q}\left(x, k_{\perp}\right)=2 \mathcal{N}_{q}(x) h\left(k_{\perp}\right) f_{q}\left(x, k_{\perp}\right),
$$

where $\mathcal{N}_{q}(x)$ is taken as a binomial function of $x$,

$$
\mathcal{N}_{q}(x)=\mathcal{N}_{q} x^{\alpha_{q}}(1-x)^{\beta_{q}}\left(\alpha_{q}+\beta_{q}\right)^{\alpha_{q}+\beta_{q}} / \alpha_{q}^{\alpha_{q}} \beta_{q}^{\beta_{q}},
$$

and $h\left(k_{\perp}\right)$ is taken as a Gaussian,

$$
h\left(k_{\perp}\right)=\sqrt{2 \mathrm{e}}\left(\left|\vec{k}_{\perp}\right| / M_{1}\right) \mathrm{e}^{-\vec{k}_{\perp}^{2} / M_{1}^{2}} .
$$

Here the Sivers function $\Delta^{N} f_{q}\left(x, k_{\perp}\right)$ is defined via

$$
f_{q / N^{\uparrow}}\left(x, k_{\perp}\right)=f_{q / N}\left(x, k_{\perp}\right)+\frac{1}{2} \Delta^{N} f_{q}\left(x, k_{\perp}\right) \vec{S} \cdot\left(\hat{p} \times \hat{k}_{\perp}\right),
$$

which is related to the Sivers function $f_{1 T}^{\perp}\left(x, k_{\perp}\right)$ defined in Eq. (3.5) by

$$
\Delta^{N} f_{q}\left(x, k_{\perp}\right)=-\frac{2\left|\vec{k}_{\perp}\right|}{M} f_{1 T}^{\perp q}\left(x, k_{\perp}\right) .
$$

There already exist different sets such as the Bochum [101-103], Torino [96, 104, 106], and Vogelsang-Yuan [105] fits. One thing seems to be clear: the Sivers function is nonzero for protons, and it has different signs for $u$ and $d$ quarks, as shown in Fig. 2 .

(3) Transversity and Collins function: A simultaneous extraction of the transversity and Collins function from SIDIS data on Collins asymmetry obtained by the HERMES [67-70] and COMPASS [71-77] collaborations and from $e^{+} e^{-}$data obtained by Belle [85-87] has been carried out by the Torino group [97, 107]. A form similar to that of the Sivers function has been taken, e.g.,
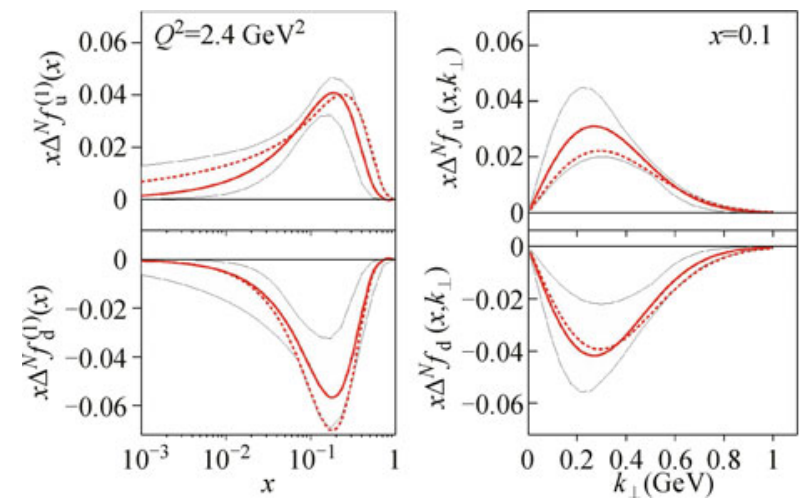

Fig. 2 Example of the parameterizations of the Sivers functions for $u$ and $d$ flavors at $Q^{2}=2.4(\mathrm{GeV} / \mathrm{c})^{2}$ by the Torino group. The figure is taken from [104]. 


$$
\begin{aligned}
& \Delta_{T} q\left(x, k_{\perp}\right)=\frac{1}{2} \mathcal{N}_{q}^{T}(x)\left[f_{q}(x)+\Delta q(x)\right] \\
& \times \mathrm{e}^{-\vec{k}_{\perp}^{2} /\left\langle\vec{k}_{\perp}^{2}\right\rangle_{T}} / \pi\left\langle\vec{k}_{\perp}^{2}\right\rangle, \\
& \Delta^{N} D_{h / q}\left(z, p_{h T}\right)=2 \mathcal{N}_{q}^{C}(z) D_{h / q}(z) h\left(p_{h T}\right) \\
& \times \mathrm{e}^{-\vec{p}_{h T}^{2} /\left\langle\vec{p}_{h T}^{2}\right\rangle} / \pi\left\langle\vec{p}_{h T}^{2}\right\rangle, \\
& \mathcal{N}_{q}^{T}(x)=\mathcal{N}_{q}^{T} x^{\alpha}(1-x)^{\beta} \frac{(\alpha+\beta)^{\alpha+\beta}}{\alpha^{\alpha} \beta^{\beta}}, \\
& \mathcal{N}_{q}^{C}(z)=\mathcal{N}_{q}^{C} z^{\gamma}(1-z)^{\delta} \frac{(\gamma+\delta)^{\gamma+\delta}}{\gamma^{\gamma} \delta^{\delta}} . \\
& h\left(p_{h T}\right)=\sqrt{2 \mathrm{e}} \frac{\left|\vec{p}_{h T}\right|}{M_{h}} \mathrm{e}^{-\vec{p}_{h T}^{2} / M_{h}^{2}},
\end{aligned}
$$

and it has been determined that the Collins function is nonzero and has different signs, e.g., for $u \rightarrow \pi^{+}$or $d \rightarrow \pi^{+}$, as shown in Fig. 3. Here, similar to the case for the Sivers function, the Collins function $\Delta^{N} D_{h / q}\left(z, k_{F \perp}\right)$ is defined via

$$
\begin{aligned}
& D_{h / q^{\uparrow}}\left(z, p_{h T}\right)=D_{q / N}\left(z, p_{h T}\right) \\
& \quad+\frac{1}{2} \Delta^{N} D_{h / q}\left(z, p_{h T}\right) \vec{s}_{q}\left(\hat{k}_{q} \times \hat{p}_{h T}\right),
\end{aligned}
$$

which is related to the Collins function $H_{1}^{\perp}\left(z, p_{h T}\right)$ defined in Eq. (3.16) by

$$
\Delta^{N} D_{h / q}\left(z, p_{h T}\right)=\frac{2\left|\vec{p}_{h T}\right|}{z M_{h}} H_{1}^{\perp q}\left(z, p_{h T}\right) .
$$

(4) Boer-Mulders function: It was pointed out [111] that the HERMES and COMPASS data on $\langle\cos 2 \phi\rangle$ asymmetry $[70,78]$ provide the first experimental evidence of the Boer-Mulders effect in SIDIS. Studies in this direction have been made in Refs. $[110,111]$ to extract the Boer-Mulders function from the SIDIS data $[70,78]$ and in Refs. $[108,109,112]$ to extract it from the Drell-Yan data [90-95]. A fit to the first moments of the Boer-Mulders functions of the $u$ and $d$ quarks is shown in Fig. 4. The form is again similar to the Sivers function, being the Sivers function just multiplied by a
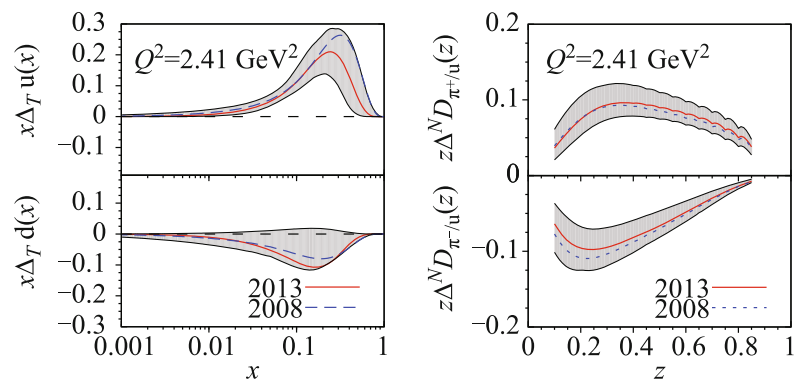

Fig. 3 Example of the Torino parameterizations of the transversity and Collins function. In the left panel, we see the transversities $x \Delta_{T} q(x)=x h_{1 q}(x)$ for $q=u, d$; in the right panel, we see the first moments of the favored and disfavored Collins functions. The figure is taken from Ref. [107].
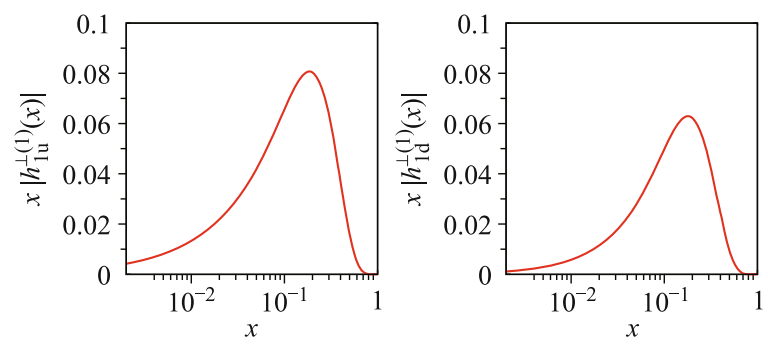

Fig. 4 First extractions of the Boer-Mulders function $h_{1}^{\perp u}(x)$ and $h_{1}^{\perp d}(x)$. This figure is taken from Ref. [111].

constant, e.g.,

$$
h_{1}^{\perp q}\left(x, k_{\perp}\right)=\lambda_{q} f_{1 T}^{\perp q}\left(x, k_{\perp}\right) .
$$

However, we note that the $\langle\cos 2 \phi\rangle$ asymmetry receives twist- 4 contributions because of the Cahn effect [22]. A proper treatment of these twist-4 effects involves twist-4 TMDs, as shown in Eq. (4.39) and in Ref. [34]. Because of the multiple gluon scattering shown in Fig. 1, the twist-4 effects could differ greatly from that given in Ref. [22], the results in which correspond to the case of $\mathcal{L}=1$. A careful check might change the conclusion obtained in Refs. [108-112].

Attempts to parameterize other TMDs such as the pretzelocity $h_{1 T}^{\perp}$ have also been made [113]. Although there is not enough data to yield high-accuracy constraints, the obtained qualitative features are also interesting.

The second part concerns the QCD evolution of the TMDs. As mentioned earlier, this is a topic that has recently developed very rapidly. Recent dedicated publications include [50-63]. QCD evolution equations have been constructed, in particular for unpolarized TMD PDFs and also for polarized TMDs such as the Sivers function. The numerical results obtained from the evolution equations show explicitly that QCD evolution is

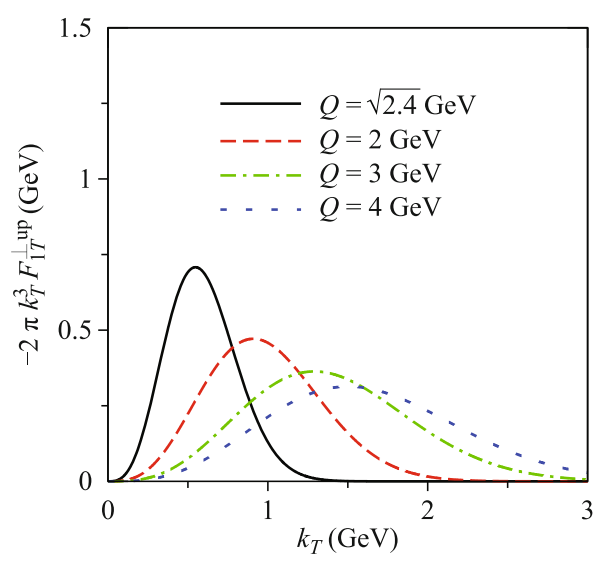

Fig. 5 Example showing the TMD evolution of the Gaussian parameterization in the low $k_{\perp}$-region. The curves show the evolved Bochum Gaussian fits of up quark Sivers function at $x=0.1$. This figure is taken from Ref. [54]. 

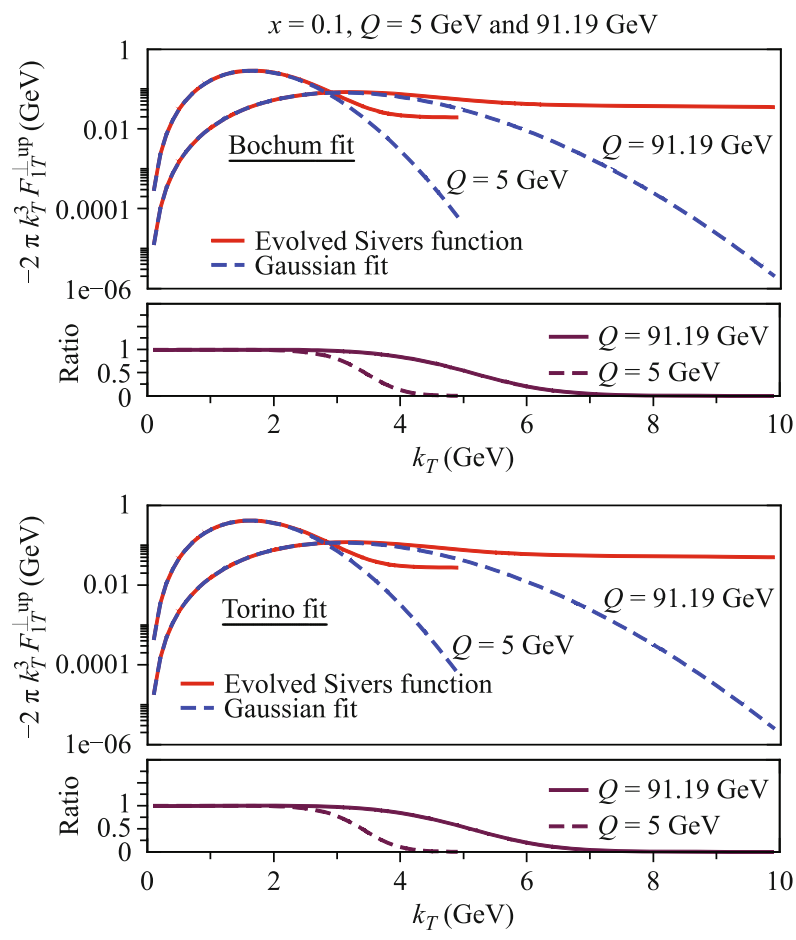

Fig. 6 Example showing the evolved $k_{\perp}$ dependence in the large $k_{\perp}$ region. Here we see the up-quark Sivers function at $Q=5 \mathrm{GeV}$ and $Q=91.19$ compared with the corresponding Gaussian fits at low- $k_{\perp}$ region at $x=0.1$. This figure is taken from Ref. [54].
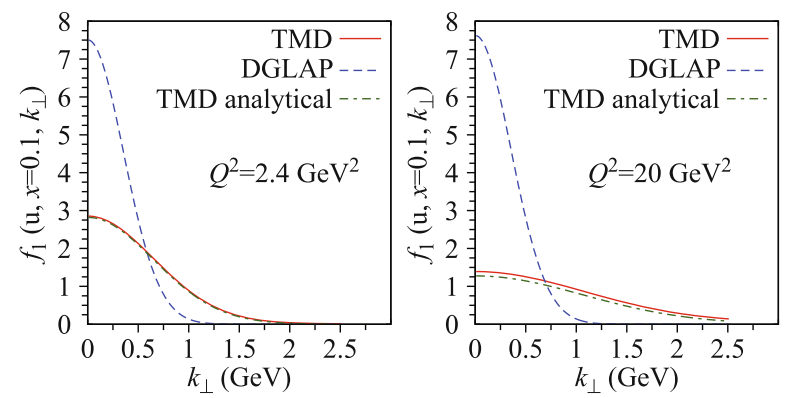

Fig. 7 Example showing the difference between the results of the TMD evolution with a DGLAP evolution for $x$-dependence only for unpolarized TMD PDF. This figure is taken from Ref. [55].

very significant for the TMDs. Not only the form of the $k_{\perp}$ dependence, but also the width of the Gaussian, evolves with $Q$. More precisely, at small $k_{\perp}$, Gaussian parameterization can be used, but the width evolves with $Q$. At larger $k_{\perp}$, the form of the $k_{\perp}$ dependence is determined mainly by gluon radiation and deviates greatly from a Gaussian; it also evolve with $Q$. In Fig. 5, we see an example for the evolution of the Gaussian parameterization at small $k_{\perp}$; in Fig. 6 , we see the evolution of the shape at large $k_{\perp}$. It is also important to use the comprehensive TMD evolution rather than a separate evolution of the transverse and longitudinal dependences. We show an example in Fig. 7.

The last thing we would like to mention regarding
TMD parameterizations is the TMD library (TMDlib). We are happy to see that an initial version was created [114] in 2014 and that it was updated recently.

\section{Summary and outlook}

In summary, by comparing the studies with what we did in constructing the theoretical framework in studying one-dimensional imaging of the nucleon with inclusive DIS, we presented a brief overview of available results on the studies of three-dimensional imaging of the nucleon with SIDIS and other semi-inclusive reactions. We summarized in particular the general form of the TMDs defined via quark-quark correlators for both TMD PDFs and FFs. We emphasized the theoretical framework for semi-inclusive reactions at LO pQCD but with leading and higher twist contributions consistently. This theoretical framework was obtained by applying the collinear expansion technique developed in the 1980s for inclusive DIS to these semi-inclusive processes. We summarized in particular how it applies to all high energy processes involving one hadron. The results obtained in such a framework should be used as starting points for studying TMDs experimentally.

Finally, we emphasize that three-dimensional imaging of the nucleon has been a hot and rapidly developing topic in recent years. Many advances have been made, and many questions are open. We see in particular that frameworks at LO pQCD and leading and higher twists for processes involving one hadron can be constructed using collinear expansions. A factorization theorem for leading twist but with LO and higher-order pQCD contributions and QCD evolution equations for unpolarized TMD PDFs and the Sivers functions have also been established. Especially in view of the operational and planned facilities such as electron-ion colliders, we expect even more rapid development in coming years.

This overview is far from complete. We apologize for omitting many aspects, such as the generalized parton distributions, the Wigner function, model calculations of TMDs, the nuclear dependences, and the hyperon polarization.

Acknowledgements We thank X. N. Wang, Y. K. Song, J. H. Gao, and many other people for collaboration and help in preparing this review. Z. T. Liang thanks also John Collins and Zebo Tang for communications. This work was supported in part by the National Natural Science Foundation of China (Grant Nos. 11035003 and 11375104), the Major State Basic Research Development Program in China (Grant No. 2014CB845406) and the CAS Center for Excellence in Particle Physics (CCEPP).

Open Access This article is distributed under the terms of the 
Creative Commons Attribution License which permits any use, distribution, and reproduction in any medium, provided the original author(s) and the source are credited.

\section{References and notes}

1. For a recent review of experiments, see e.g., A. D. Krisch, Hard collisions of spinning protons: Past, present and future, Eur. Phys. J. A 31(4), 417 (2007), there are also a number of reviews on this topic, see e.g., Z. T. Liang and C. Boros, Int. J. Mod. Phys. A 15, 927 (2000), V. Barone, F. Bradamante, and A. Martin, Transverse-spin and transverse-momentum effects in high-energy processes, Prog. Part. Nucl. Phys. 65(2), 267 (2010)

2. Z. T. Liang, Three dimensional imaging of the nucleon-TMD (theory and phenomenology), arXiv: 1502.03896 [hep-ph], Plenary talk at the 21st International Symposium on Spin Physics, October 20-24, 2014

3. D. Boer, Overview of TMD evolution, arXiv: 1502.00899 [hep-ph], Invited talk at the 21st International Symposium on Spin Physics, October 20-24, 2014

4. J. Collins, TMD theory, factorization and evolution, Int. J. Mod. Phys. Conf. Ser. 25, 1460001 (2014), arXiv: 1307.2920

5. There are definitely cases where factorization might break down, see e.g., J. P. Ma, J. X. Wang, and S. Zhao, Breakdown of QCD factorization for P-wave quarkonium production at low transverse momentum, Phys. Lett. B 737, 103 (2014), arXiv: 1405.3373 [hep-ph], and the references given there

6. J. C. Collins, D. E. Soper, and G. F. Sterman, Factorization of hard processes in QCD, Adv. Ser. Direct. High Energy Phys. 5, 1 (1989), arXiv: hep-ph/0409313

7. J. Collins, Foundations of perturbative QCD (Cambridge monographs on particle physics, nuclear physics and cosmology), Cambridge: Cambridge University Press, 2011

8. R. P. Feynman, Photon Hadron Interactions, W. A. Benjamin, 1972

9. R. K. Ellis, W. Furmanski, and R. Petronzio, Power corrections to the parton model in QCD, Nucl. Phys. B 207(1), 1 (1982)

10. R. K. Ellis, W. Furmanski, and R. Petronzio, Unravelling higher twists, Nucl. Phys. B 212(1), 29 (1983)

11. J. W. Qiu and G. F. Sterman, Power corrections in hadronic scattering (I): Leading $1 / Q^{2}$ corrections to the Drell-Yan cross section, Nucl. Phys. B 353(1), 105 (1991); J. W. Qiu and G. F. Sterman, Power corrections in hadronic scattering (II): Factorization, Nucl. Phys. B 353(1), 137 (1991)

12. G. L. Kane, J. Pumplin, and W. Repko, Transverse quark polarization in large- $p_{T}$ reactions, $\mathrm{e}^{+} \mathrm{e}^{-}$jets, and leptoproduction: A test of quantum chromodynamics, Phys. Rev. Lett. 41(25), 1689 (1978)

13. D. L. Adams, et al. (FNAL-E704 Collaboration), Analyzing power in inclusive $\pi^{+}$and $\pi^{-}$production at high $x_{F}$ with a $200 \mathrm{GeV}$ polarized proton beam, Phys. Lett. B 264(3-4), 462 (1991)

14. D. W. Sivers, Single-spin production asymmetries from the hard scattering of pointlike constituents, Phys. Rev. D 41(1), 83 (1990); D. W. Sivers, Hard-scattering scaling laws for single-spin production asymmetries, Phys. Rev. D 43(1), 261 (1991)

15. C. Boros, Z. T. Liang, and T. C. Meng, Quark spin distribution and quark-antiquark annihilation in single-spin hadronhadron collisions, Phys. Rev. Lett. 70(12), 1751 (1993)

16. J. C. Collins, Fragmentation of transversely polarized quarks probed in transverse momentum distributions, Nucl. Phys. B 396(1), 161 (1993)

17. S. J. Brodsky, D. S. Hwang, and I. Schmidt, Final-state interactions and single-spin asymmetries in semi-inclusive deep inelastic scattering, Phys. Lett. B 530(1-4), 99 (2002)

18. J. C. Collins, Leading-twist single-transverse-spin asymmetries: Drell-Yan and deep-inelastic scattering, Phys. Lett. B 536(1-2), 43 (2002)

19. X. Ji and F. Yuan, Parton distributions in light-cone gauge: Where are the final-state interactions? Phys. Lett. B 543(12), 66 (2002)

20. A. V. Belitsky, X. Ji, and F. Yuan, Final state interactions and gauge invariant parton distributions, Nucl. Phys. B 656(1-2), 165 (2003)

21. H. Georgi and H. Politzer, Clean tests of quantum chromodynamics in $\mu p$ scattering, Phys. Rev. Lett. 40(1), 3 (1978)

22. R. N. Cahn, Azimuthal dependence in leptoproduction: A simple parton model calculation, Phys. Lett. B 78, 269 (1978)

23. K. Goeke, A. Metz, and M. Schlegel, Parameterization of the quark-quark correlator of a spin- $1 / 2$ hadron, Phys. Lett. B 618(1-4), 90 (2005)

24. P. Mulders, invited talk at the 21st International Symposium on Spin Physics, October 20-24, 2014, Beijing, China, and lectures in 17th Taiwan nuclear physics summer school, Aug. 25-28, 2014

25. A. Bacchetta and P. J. Mulders, Deep inelastic leptoproduction of spin-one hadrons, Phys. Rev. D 62(11), 114004 (2000), arXiv: hep-ph/0007120

26. K. B. Chen, S. Y. Wei, W. H. Yang, and Z. T. Liang, Three dimensional fragmentation functions from the quark-quark correlator, arXiv: 1505.02856 [hep-ph]

27. M. Gourdin, Semi-inclusive reactions induced by leptons, Nucl. Phys. B 49, 501 (1972)

28. A. Kotzinian, New quark distributions and semi-inclusive electroproduction on polarized nucleons, Nucl. Phys. B 441(1-2), 234 (1995)

29. M. Diehl and S. Sapeta, On the analysis of lepton scattering on longitudinally or transversely polarized protons, Eur. Phys. J. C 41(4), 515 (2005)

30. A. Bacchetta, M. Diehl, K. Goeke, A. Metz, P. J. Mulders and M. Schlegel, Semi-inclusive deep inelastic scattering at 
small transverse momentum, J. High Energy Phys. 0702, 093 (2007)

31. S. Arnold, A. Metz, and M. Schlegel, Dilepton production from polarized hadron hadron collisions, Phys. Rev. D 79(3), 034005 (2009)

32. D. Pitonyak, M. Schlegel, and A. Metz, Polarized hadron pair production from electron-positron annihilation, Phys. Rev. D 89(5), 054032 (2014)

33. Z. Liang and X. N. Wang, Azimuthal and single-spin asymmetry in deep-inelastic lepton-nucleon scattering, Phys. Rev. D 75(9), 094002 (2007)

34. Y. Song, J. Gao, Z. T. Liang, and X. N. Wang, Twist-4 contributions to the azimuthal asymmetry in semi-inclusive deeply inelastic scattering, Phys. Rev. D 83(5), 054010 (2011)

35. Y. Song, J. Gao, Z. T. Liang, and X. N. Wang, Azimuthal asymmetries in semi-inclusive deep inelastic scattering with polarized beam and/or target and their nuclear dependences, Phys. Rev. D 89(1), 014005 (2014)

36. S. y. Wei, Y. Song, and Z. Liang, Higher-twist contribution to fragmentation function in inclusive hadron production in $\mathrm{e}^{+} \mathrm{e}^{-}$annihilation, Phys. Rev. D 89(1), 014024 (2014)

37. S. Y. Wei, K. Chen, Y. Song, and Z. Liang, Leading and higher twist contributions in semi-inclusive $\mathrm{e}^{+} \mathrm{e}^{-}$annihilation at high energies, Phys. Rev. D 91(3), 034015 (2015), arXiv: 1410.4314 [hep-ph]

38. P. J. Mulders and R. D. Tangerman, The complete tree-level result up to order $1 / Q$ for polarized deep-inelastic leptoproduction, Nucl. Phys. B 461(1-2), 197 (1996) [Erratum, Nucl. Phys. B 484, 538 (1997)]

39. D. Boer, R. Jakob, and P. J. Mulders, Asymmetries in polarized hadron production in $\mathrm{e}^{+} \mathrm{e}^{-}$annihilation up to order 1/Q, Nucl. Phys. B 504(1-2), 345 (1997)

40. Z. Lu and I. Schmidt, Transverse momentum dependent twist-three result for polarized Drell-Yan processes, Phys. Rev. D 84(11), 114004 (2011)

41. A. P. Chen, J. P. Ma, and G. P. Zhang, Twist-3 contributions in semi-inclusive DIS with transversely polarized target, arXiv: 1505.03217 [hep-ph]

42. J. C. Collins and D. E. Soper, Back-to-back jets in QCD, Nucl. Phys. B 193(2), 381 (1981) [Erratum, Nucl. Phys. B 213, 545 (1983)]

43. J. C. Collins and D. E. Soper, Parton distribution and decay functions, Nucl. Phys. B 194(3), 445 (1982)

44. J. C. Collins, D. E. Soper, and G. F. Sterman, Transverse momentum distribution in Drell-Yan pair and W and Z boson production, Nucl. Phys. B 250(1-4), 199 (1985)

45. J. C. Collins, D. E. Soper, and G. F. Sterman, Factorization for short distance hadron-hadron scattering, Nucl. Phys. B 261, 104 (1985)

46. X. Ji, J. P. Ma, and F. Yuan, Factorization of large-x quark distributions in a hadron, Phys. Lett. B 610(3-4), 247 (2005)
47. A. Idilbi, X. Ji, J. P. Ma, and F. Yuan, Collins-Soper equation for the energy evolution of transverse-momentum and spin dependent parton distributions, Phys. Rev. D 70(7), 074021 (2004)

48. X. Ji, J. P. Ma, and F. Yuan, QCD factorization for spindependent cross sections in DIS and Drell-Yan processes at low transverse momentum, Phys. Lett. B 597(3-4), 299 (2004)

49. X. Ji, J. Ma, and F. Yuan, QCD factorization for semiinclusive deep-inelastic scattering at low transverse momentum, Phys. Rev. D 71(3), 034005 (2005)

50. A. A. Henneman, D. Boer, and P. J. Mulders, Evolution of transverse momentum dependent distribution and fragmentation functions, Nucl. Phys. B 620(1-2), 331 (2002)

51. J. Zhou, F. Yuan, and Z. T. Liang, QCD evolution of the transverse momentum dependent correlations, Phys. Rev. D 79(11), 114022 (2009)

52. Z. B. Kang, B. W. Xiao, and F. Yuan, QCD resummation for single spin asymmetries, Phys. Rev. Lett. 107(15), 152002 (2011)

53. S. M. Aybat and T. C. Rogers, Transverse momentum dependent parton distribution and fragmentation functions with QCD evolution, Phys. Rev. D 83(11), 114042 (2011)

54. S. M. Aybat, J. C. Collins, J. W. Qiu, and T. C. Rogers, QCD evolution of the Sivers function, Phys. Rev. D 85(3), 034043 (2012)

55. M. Anselmino, M. Boglione, and S. Melis, Strategy towards the extraction of the Sivers function with transverse momentum dependent evolution, Phys. Rev. D 86(1), 014028 (2012)

56. P. Sun and F. Yuan, Transverse momentum dependent evolution: Matching semi-inclusive deep inelastic scattering processes to Drell-Yan and W/Z boson production, Phys. Rev. D 88(11), 114012 (2013)

57. J. P. Ma and G. P. Zhang, QCD corrections of all structure functions in transverse momentum dependent factorization for Drell-Yan processes, J. High Energy Phys. 2014(2), 100 (2014), arXiv: 1308.2044 [hep-ph]

58. M. G. Echevarria, A. Idilbi, Z. B. Kang, and I. Vitev, QCD evolution of the Sivers asymmetry, Phys. Rev. D 89(7), 074013 (2014)

59. C. A. Aidala, B. Field, L. P. Gamberg, and T. C. Rogers, Limits on transverse momentum dependent evolution from semi-inclusive deep inelastic scattering at moderate $Q$, Phys. Rev. D 89(9), 094002 (2014)

60. Z. B. Kang, A. Prokudin, P. Sun, and F. Yuan, Nucleon tensor charge from Collins azimuthal asymmetry measurements, Phys. Rev. D 91(7), 071501 (2015), arXiv: 1410.4877 [hep-ph]

61. M. G. Echevarria, A. Idilbi, and I. Scimemi, Unified treatment of the QCD evolution of all (un-)polarized transverse momentum dependent functions: Collins function as a study case, Phys. Rev. D 90(1), 014003 (2014) 
62. J. Collins and T. Rogers, Understanding the large-distance behavior of transverse-momentum-dependent parton densities and the Collins-Soper evolution kernel, Phys. Rev. D 91(7), 074020 (2015), arXiv: 1412.3820 [hep-ph]

63. Z. B. Kang, A. Prokudin, P. Sun, and F. Yuan, Extraction of quark transversity distribution and Collins fragmentation functions with QCD evolution, arXiv: 1505.05589 [hep-ph]

64. A. Rostomyan (for the HERMES collaboration), "Highlights of HERMES", Plenary talk at the 21st International Symposium on Spin Physics, October 20-24, 2014, Beijing, China

65. M. Stolarski (On behalf of the COMPASS Collaboration), "Latest results from the COMPASS experiment", Plenary talk at the 21st International Symposium on Spin Physics, October 20-24, 2014, Beijing, China

66. A. Airapetian, et al. (HERMES Collaboration), Evidence for a single-spin azimuthal asymmetry in semi-inclusive Pion electroproduction, Phys. Rev. Lett. 84(18), 4047 (2000), arXiv: hep-ex/9910062

67. A. Airapetian, et al. (HERMES Collaboration), Single-spin asymmetries in semi-inclusive deep-inelastic scattering on a transversely polarized hydrogen target, Phys. Rev. Lett. 94(1), 012002 (2005), arXiv: hep-ex/0408013

68. A. Airapetian, et al. (HERMES Collaboration), Observation of the naive- $T$-odd Sivers effect in deep-inelastic scattering, Phys. Rev. Lett. 103(15), 152002 (2009), arXiv: 0906.3918 [hep-ex]

69. A. Airapetian, et al. (HERMES Collaboration), Effects of transversity in deep-inelastic scattering by polarized protons, Phys. Lett. B 693(1), 11 (2010), arXiv: 1006.4221 [hep$\mathrm{ex}]$

70. A. Airapetian, et al. (HERMES Collaboration), Azimuthal distributions of charged hadrons, pions, and kaons produced in deep-inelastic scattering off unpolarized protons and deuterons, Phys. Rev. D 87(1), 012010 (2013), arXiv: 1204.4161 [hep-ex]

71. V. Y. Alexakhin, et al. (COMPASS Collaboration), First measurement of the transverse spin asymmetries of the deuteron in semi-inclusive deep inelastic scattering, Phys. Rev. Lett. 94(20), 202002 (2005), arXiv: hep-ex/0503002

72. E. S. Ageev, et al. (COMPASS Collaboration), A new measurement of the Collins and Sivers asymmetries on a transversely polarised deuteron target, Nucl. Phys. B 765(1-2), 31 (2007), arXiv: hep-ex/0610068

73. M. Alekseev, et al. (COMPASS Collaboration), Collins and Sivers asymmetries for pions and kaons in muon-deuteron DIS, Phys. Lett. B 673(2), 127 (2009), arXiv: 0802.2160 [hep-ex]

74. M. G. Alekseev, et al. (COMPASS Collaboration), Measurement of the Collins and Sivers asymmetries on transversely polarised protons, Phys. Lett. B 692(4), 240 (2010), arXiv: 1005.5609 [hep-ex]

75. C. Adolph, et al. (COMPASS Collaboration), I - Experimental investigation of transverse spin asymmetries in $\mu$-p
SIDIS processes: Collins asymmetries, Phys. Lett. B 717, 376 (2012), arXiv: 1205.5121 [hep-ex]

76. C. Adolph, et al. (COMPASS Collaboration), II - Experimental investigation of transverse spin asymmetries in $\mu$-p SIDIS processes: Sivers asymmetries, Phys. Lett. B 717, 383 (2012), arXiv: 1205.5122 [hep-ex]

77. C. Adolph, et al. (COMPASS Collaboration), Collins and Sivers asymmetries in muonproduction of pions and kaons off transversely polarised protons, Phys. Lett. B 744, 250 (2015), arXiv: 1408.4405 [hep-ex]

78. C. Adolph, et al. (COMPASS Collaboration), Measurement of azimuthal hadron asymmetries in semi-inclusive deep inelastic scattering off unpolarised nucleons, Nucl. Phys. B 886, 1046 (2014), arXiv: 1401.6284 [hep-ex]

79. H. Avakian, et al. (The CLAS Collaboration), Measurement of single- and double-spin asymmetries in deep inelastic pion electroproduction with a longitudinally polarized target, Phys. Rev. Lett. 105(26), 262002 (2010), arXiv: 1003.4549 [hep-ex]

80. M. Aghasyan, H. Avakian, P. Rossi, E. De Sanctis, D. Hasch, M. Mirazita, D. Adikaram, M. J. Amaryan, et al., Precise measurements of beam spin asymmetries in semi-inclusive $\pi^{0}$ production, Phys. Lett. B 704, 397 (2011), arXiv: 1106.2293 [hep-ex]

81. X. Qian, et al. (Jefferson Lab Hall A Collaboration), Single spin asymmetries in charged Pion production from semiinclusive deep inelastic scattering on a transversely polarized $\mathrm{He}^{3}$ target at $Q^{2}=1.4-2.7 \mathrm{GeV}^{2}$, Phys. Rev. Lett. 107(7), 072003 (2011), arXiv: 1106.0363 [nucl-ex]

82. J. Huang, et al. (Jefferson Lab Hall A Collaboration), Beamtarget double-spin asymmetry ALT in charged pion production from deep inelastic scattering on a transversely polarized $\mathrm{He}^{3}$ target at $1.4<Q^{2}<2.7 \mathrm{GeV}^{2}$, Phys. Rev. Lett. 108(5), 052001 (2012), arXiv: 1108.0489 [nucl-ex]

83. Y. Zhang, et al. (Jefferson Lab Hall A Collaboration), Measurement of "pretzelosity" asymmetry of charged pion production in semi-inclusive deep inelastic scattering on a polarized $\mathrm{He}^{3}$ target, Phys. Rev. C 90(5), 055209 (2014), arXiv: 1312.3047 [nucl-ex]

84. Y. X. Zhao, et al. (Jefferson Lab Hall A Collaboration), Single spin asymmetries in charged kaon production from semiinclusive deep inelastic scattering on a transversely polarized $\mathrm{He}^{3}$ target, Phys. Rev. C 90(5), 055201 (2014), arXiv: 1404.7204 [nucl-ex]

85. K. Abe, et al. (Belle Collaboration), Measurement of azimuthal asymmetries in inclusive production of hadron pairs in $\mathrm{e}^{+} \mathrm{e}^{-}$annihilation at Belle, Phys. Rev. Lett. 96, 232002 (2006), arXiv: hep-ex/0507063

86. R. Seidl, et al. (Belle Collaboration), Measurement of azimuthal asymmetries in inclusive production of hadron pairs in $\mathrm{e}^{+} \mathrm{e}^{-}$annihilation at $\sqrt{s}=10.58 \mathrm{GeV}$, Phys. Rev. D 78(3), 032011 (2008) [Erratum: Phys. Rev. D 86, 039905 (2012)], arXiv: 0805.2975 [hep-ex] 
87. A. Vossen, et al. (Belle Collaboration), Observation of transverse polarization asymmetries of charged pion pairs in $\mathrm{e}^{+} \mathrm{e}^{-}$ annihilation near $\sqrt{s}=10.58 \mathrm{GeV}$, Phys. Rev. Lett. 107(7), 072004 (2011), arXiv: 1104.2425 [hep-ex]

88. J. P. Lees, et al. (BaBar Collaboration), Measurement of Collins asymmetries in inclusive production of charged pion pairs in $\mathrm{e}^{+} \mathrm{e}^{-}$annihilation at BABAR, Phys. Rev. D 90(5), 052003 (2014), arXiv: 1309.5278 [hep-ex]

89. Y. Guan, I. Garzia, H. Li, X. R. Lyu, and W. Yan, Future results on fragmentation functions from BESIII, EPJ Web Conf. 85, 02037 (2015); also talk given at the 21st International Symposium on Spin Physics, October 20-24, 2014, Beijing, China

90. J. Badier, et al. (NA3 Collaboration), Angular distributions in the dimuon hadronic production at $150 \mathrm{GeV} / \mathrm{c}$, Z. Phys. C 11, 195 (1981)

91. S. Falciano, et al. (NA10 Collaboration), Angular distributions of muon pairs produced by $194 \mathrm{GeV} / \mathrm{c}$ negative pions, Z. Phys. C 31, 513 (1986)

92. M. Guanziroli, et al. (NA10 Collaboration), Angular distributions of muon pairs produced by negative pions on deuterium and tungsten, Z. Phys. C 37, 545 (1988)

93. J. S. Conway, C. E. Adolphsen, J. P. Alexander, K. J. Anderson, J. G. Heinrich, J. E. Pilcher, A. Possoz, E. I. Rosenberg, C. Biino, J. F. Greenhalgh, W. C. Louis, K. T. McDonald, S. Palestini, F. C. Shoemaker, and A. J. S. Smith, Experimental study of muon pairs produced by $252-\mathrm{GeV}$ pions on tungsten, Phys. Rev. D 39(1), 92 (1989)

94. L. Y. Zhu, et al. (NuSea Collaboration), Measurement of angular distributions of Drell-Yan dimuons in $\mathrm{p}+\mathrm{d}$ interactions at $800 \mathrm{GeV} / \mathrm{c}$, Phys. Rev. Lett. 99(8), 082301 (2007), arXiv: hep-ex/0609005

95. L. Y. Zhu, et al. (NuSea Collaboration), Measurement of angular distributions of Drell-Yan dimuons in $\mathrm{p}+\mathrm{p}$ interactions at $800 \mathrm{GeV} / \mathrm{c}$, Phys. Rev. Lett. 102(18), 182001 (2009), arXiv: 0811.4589 [nucl-ex]

96. M. Anselmino, M. Boglione, U. D'Alesio, A. Kotzinian, F. Murgia, and A. Prokudin, Role of Cahn and Sivers effects in deep inelastic scattering, Phys. Rev. D 71(7), 074006 (2005)

97. M. Anselmino, M. Boglione, U. D'Alesio, A. Kotzinian, F. Murgia, A. Prokudin, and C. Turk, Transversity and Collins functions from SIDIS and $\mathrm{e}^{+} \mathrm{e}^{-}$data, Phys. Rev. D75(5), 054032 (2007)

98. P. Schweitzer, T. Teckentrup, and A. Metz, Intrinsic transverse parton momenta in deeply inelastic reactions, Phys. Rev. D 81(9), 094019 (2010)

99. A. Signori, A. Bacchetta, M. Radici, and G. Schnell, Investigations into the flavor dependence of partonic transverse momentum, J. High Energy Phys. 2013(11), 194 (2013)

100. M. Anselmino, M. Boglione, J. O. Gonzalez, S. Melis, and A. Prokudin, Unpolarised transverse momentum dependent distribution and fragmentation functions from SIDIS multiplicities, J. High Energy Phys. 1404, 005 (2014), arXiv: 1312.6261

101. A. V. Efremov, K. Goeke, S. Menzel, A. Metz, and P. Schweitzer, Sivers effect in semi-inclusive DIS and in the Drell-Yan process, Phys. Lett. B 612(3-4), 233 (2005)

102. J. C. Collins, A. V. Efremov, K. Goeke, S. Menzel, A. Metz, and P. Schweitzer, Sivers effect in semiinclusive deeply inelastic scattering, Phys. Rev. D 73(1), 014021 (2006)

103. S. Arnold, A. V. Efremov, K. Goeke, M. Schlegel, and P. Schweitzer, Sivers effect at Hermes, Compass and Clas12, arXiv: 0805.2137 [hep-ph]

104. M. Anselmino, M. Boglione, U. D'Alesio, A. Kotzinian, S. Melis, F. Murgia, A. Prokudin, and C. Turk, Sivers effect for pion and kaon production in semi-inclusive deep inelastic scattering, Eur. Phys. J. A 39(1), 89 (2009)

105. W. Vogelsang and F. Yuan, Single-transverse-spin asymmetries: From deep inelastic scattering to hadronic collisions, Phys. Rev. D 72(5), 054028 (2005)

106. A. Bacchetta and M. Radici, Constraining quark angular momentum through semi-inclusive measurements, Phys. Rev. Lett. 107(21), 212001 (2011)

107. M. Anselmino, M. Boglione, U. D'Alesio, S. Melis, F. Murgia, and A. Prokudin, Simultaneous extraction of transversity and Collins functions from new semi-inclusive deep inelastic scattering and $\mathrm{e}^{+} \mathrm{e}^{-}$data, Phys. Rev. D 87(9), 094019 (2013)

108. B. Zhang, Z. Lu, B. Q. Ma, and I. Schmidt, Extracting BoerMulders functions from $\mathrm{p}+\mathrm{D}$ Drell-Yan processes, Phys. Rev. D 77(5), 054011 (2008)

109. B. Zhang, Z. Lu, B. Q. Ma, and I. Schmidt, $\cos 2 \phi$ asymmetries in unpolarized semi-inclusive DIS, Phys. Rev. D 78(3), 034035 (2008)

110. V. Barone, A. Prokudin, and B. Q. Ma, Systematic phenomenological study of the $\cos 2 \phi$ asymmetry in unpolarized semi-inclusive DIS, Phys. Rev. D 78(4), 045022 (2008)

111. V. Barone, S. Melis, and A. Prokudin, Boer-Mulders effect in unpolarized SIDIS: An analysis of the COMPASS and HERMES data on the cos $2 \phi$ asymmetry, Phys. Rev. D 81(11), 114026 (2010)

112. Z. Lu and I. Schmidt, Updating Boer-Mulders functions from unpolarized pd and pp Drell-Yan data, Phys. Rev. D 81(3), 034023 (2010)

113. J. Zhu and B. Q. Ma, Probing the leading-twist transversemomentum-dependent parton distribution function $h_{1 T}^{\perp}$ via the polarized proton-antiproton Drell-Yan process, Phys. Rev. D 82(11), 114022 (2010)

114. F. Hautmann, H. Jung, M. Krer, P. J. Mulders, E. R. Nocera, T. C. Rogers, and A. Signori, TMDlib and TMDplotter: Library and plotting tools for transverse-momentumdependent parton distributions, Eur. Phys. J. C 74(12), 3220 (2014) 